\title{
Age consistency between exoplanet hosts and field stars
}

\author{
A. Bonfanti ${ }^{1,2}$, S. Ortolani ${ }^{1,2}$, and V. Nascimbeni ${ }^{2}$ \\ 1 Dipartimento di Fisica e Astronomia, Università degli Studi di Padova, Vicolo dell'Osservatorio 3, 35122 Padova, Italy \\ e-mail: andrea . bonfanti.1@studenti .unipd.it \\ 2 Osservatorio Astronomico di Padova, INAF, Vicolo dell'Osservatorio 5, 35122 Padova, Italy
}

Received 2 September 2015 / Accepted 3 November 2015

\begin{abstract}
Context. Transiting planets around stars are discovered mostly through photometric surveys. Unlike radial velocity surveys, photometric surveys do not tend to target slow rotators, inactive or metal-rich stars. Nevertheless, we suspect that observational biases could also impact transiting-planet hosts.

Aims. This paper aims to evaluate how selection effects reflect on the evolutionary stage of both a limited sample of transiting-planet host stars (TPH) and a wider sample of planet-hosting stars detected through radial velocity analysis. Then, thanks to uniform derivation of stellar ages, a homogeneous comparison between exoplanet hosts and field star age distributions is developed.

Methods. Stellar parameters have been computed through our custom-developed isochrone placement algorithm, according to Padova evolutionary models. The notable aspects of our algorithm include the treatment of element diffusion, activity checks in terms of $\log R_{H K}^{\prime}$ and $v \sin i$, and the evaluation of the stellar evolutionary speed in the Hertzsprung-Russel diagram in order to better constrain age. Working with TPH, the observational stellar mean density $\rho_{\star}$ allows us to compute stellar luminosity even if the distance is not available, by combining $\rho_{\star}$ with the spectroscopic $\log g$.

Results. The median value of the TPH ages is $\sim 5$ Gyr. Even if this sample is not very large, however the result is very similar to what we found for the sample of spectroscopic hosts, whose modal and median values are [3, 3.5) Gyr and 4.8 Gyr, respectively. Thus, these stellar samples suffer almost the same selection effects. An analysis of MS stars of the solar neighbourhood belonging to the same spectral types bring to an age distribution similar to the previous ones and centered around solar age value. Therefore, the age of our Sun is consistent with the age distribution of solar neighbourhood stars with spectral types from late $\mathrm{F}$ to early $\mathrm{K}$, regardless of whether they harbour planets or not. We considered the possibility that our selected samples are older than the average disc population.
\end{abstract}

Key words. stars: evolution - Hertzsprung-Russell and C-M diagrams - planetary systems

\section{Introduction}

Computing ages of field stars is very challenging because the age is not a direct observable. Thanks to models, information about the age comes from the composition and evolutionary state of the core of a star, while we are mostly limited to observing the properties at the surface. Several techniques can be applied.

Using the stellar effective temperature $T_{\text {eff }}$ and luminosity $L$ as input values, age can be computed through interpolation in the grids of isochrones (isochrone placement). Instead, gyrochronology (see e.g. Barnes \& Kim 2010) is an empirical technique that allows the determination of stellar ages considering that the rotational speed of stars declines with time because of magnetic braking. Asteroseismology (see Handler 2013 for a review) is a very promising technique because the individual oscillation frequencies are directly linked to the inner density profile and the sound propagation speed in the stellar core. These frequencies are recovered through detailed analyses and high-precision photometry, which facilitates the determination of very precise, though model-dependent, ages. If it is not possible to investigate each oscillation mode, asteroseismic studies simply give global parameters i.e. the large frequency separation $\Delta v$ and the frequency of maximum power $v_{\max }$, which are linked to the stellar mean density $\rho_{\star}$ and surface gravity $\log g$ (see e.g. Kjeldsen $\&$ Bedding 1995). In this case, asteroseismology loses part of its strength. Input $T_{\mathrm{eff}}, \rho_{\star}$ and $\log g$ again require isochrones to compute ages as in Chaplin et al. (2014), however, asteroseismic $\log g$ is known with better precision if compared with the spectroscopic value, for instance. For a broad review about different age computation methods, see Soderblom (2010).

Since $T_{\text {eff }}$ and $L$ can be usually recovered for many stars, in this paper we compute the ages of transiting-planet host stars (TPH) in a homogeneous way via isochrones. Knowledge of stellar ages is particularly important in the context of planethosting stars (SWP). The age distribution of SWP tells us whether planets are preferentially hosted by young or old stars. This is related to the dynamical stability of the systems and with the mutual influence between planets and hosting star; see e.g. Pätzold et al. (2004), Barker \& Ogilvie (2009), Debes \& Jackson (2010). Moreover, ages for exoplanet host stars enable a comparison with typical timescales of biological evolution and an assessment of the plausibility of the presence of life (see e.g. Kasting \& Catling 2003).

The paper is organised in the following way: Sect. 2 describes the characteristics of our stellar sample and the isochrones. Section 3 presents the central aspects of our algorithm, Sect. 4 shows the results, and Sect. 5 summarizes our work.

\section{The data}

\subsection{Planet-hosting stars catalogues}

We analysed the ages of those stars whose planets were discovered through the transit method. In principle, these kinds of 
stars should not suffer from biases: (1) the stars that are chosen are not necessarily inactive, unlike in radial velocity surveys, where spectroscopic analysis requires sharp and well-defined lines. However, we caution that it is indeed more difficult to detect transits for stars with a large amplitude of intrinsic variability. (2) These TPH stars are not necessarily slow rotators, unlike in radial velocity surveys. In fact, rotation broadens the lines and reduces their depth, making spectroscopic analysis less precise, however, once a possible transit signal is detected, spectroscopic validation is required to confirm such a planet. Therefore, stars belonging to photometric surveys must also be suitable for spectroscopic analyses if exoplanet validation is expected, so almost the same biases are expected. In fact, in the case of transiting planet hosts, there are other systematic selection effects. Transiting-planet hosts are expected to be preferentially edgeon, even if spin-orbit misalignment occurs in some exoplanetary systems. Gravity darkening or differential rotation (von Zeipel 1924; Maeder 1999) could affect stellar observables. In addition, the hosted planets are very close to their own star.

We selected 61 transiting-planet hosts from SWEET-Cat, a catalogue of stellar parameters for stars with planets ${ }^{1}$ (Santos et al. 2013), to obtain our transiting-planet hosts (TPH) catalogue. Among the stars of this catalogue, we further consider only those stars brighter than $V=12$ and this inevitably introduces a further source of bias. This criterion takes into account that future photometric missions with the aim of characterizing exoplanets, such as CHEOPS (Broeg et al. 2013) or PLATO (Rauer et al. 2014), will investigate bright stars. This led us to the Bright Transiting-Planet Hosts (BTPH) catalogue, which is composed of 43 stars. The metallicity $[\mathrm{Fe} / \mathrm{H}]$ and the logarithm of the surface gravity $\log g$ are always available from Sweet-Cat. If available, we took $V$ magnitude and $B-V$ colour index from Maxted et al. (2011), otherwise we collected $V$ from SWEET-Cat and $B-V$ from The Site of California and Carnegie Program for Extrasolar Planet Search: Exoplanets Data Explorer ${ }^{2}$. As reported by Maxted et al. (2011), the target stars of surveys that aim to discover exoplanets through transits are typically characterized by optical photometry of poor quality in the range $V=8.5-13$ mag. For stars brighter than $V \approx 12$, optical photometry is usually available from Tycho catalogue, nevertheless, this catalogue is only complete up to $V \approx 11$ and photometric accuracy rapidly deteriorates for $V \gtrsim 9.5$. The authors give high-quality photoelectric optical photometry for planet-hosting stars (mostly WASP discoveries), so we decided to use these data if available.

We also built a catalogue of 274 planet-hosting stars whose planets were detected through radial velocity method (spectroscopic hosts: SH catalogue) from SWEET-Cat.

\subsection{Solar neighbourhood catalogues}

We built a catalogue of F-G-K main sequence stars (MS-stars) belonging to the solar neighbourhood ( $\mathrm{SN}$ catalogue) by taking stellar data from the re-analysis of the Geneva-Copenhagen survey by Casagrande et al. (2011). It is a survey of late-type dwarf stars that are magnitude limited at $V \approx 8.3$; the authors computed the ages for these stars. In particular, we collected the 7044 stars with available ages, belonging to the MS. The MS containing the F-G-K stars has been indentified by selecting a strip going from $T_{\text {eff }} \approx 4500 \mathrm{~K}$ to $T_{\text {eff }} \approx 7100 \mathrm{~K}$, within a range of $0.45 \mathrm{dex}$ in $\log L$, whose minimum and maximum values are $-1.24 \mathrm{dex}$

\footnotetext{
1 https://www.astro.up.pt/resources/sweet-cat/

2 http://exoplanets.org/table
}

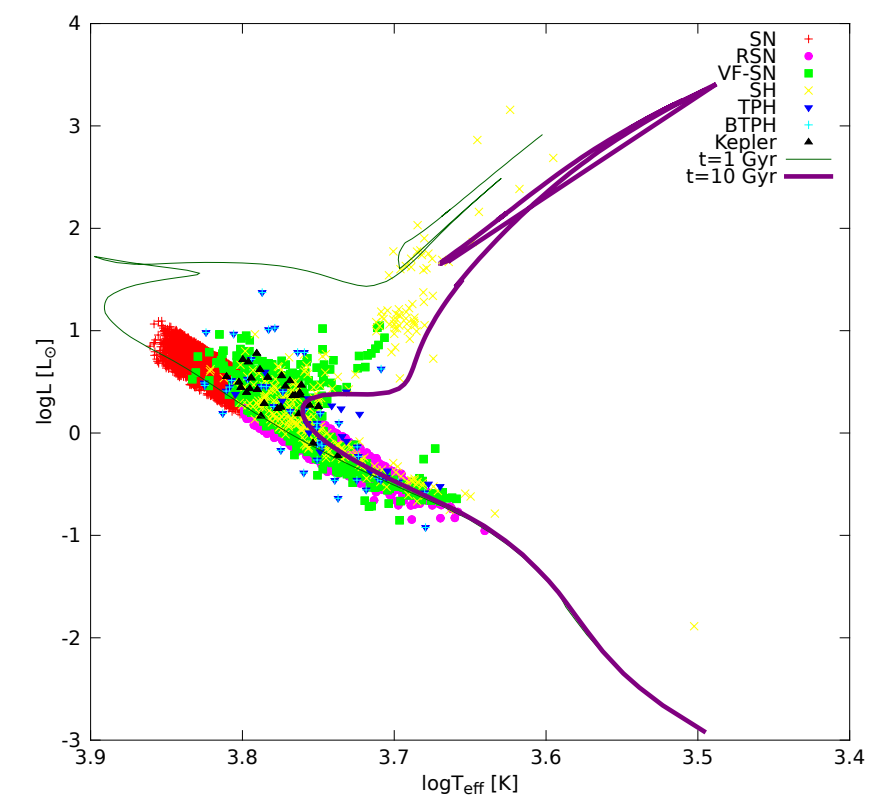

Fig. 1. Stars belonging to our custom-built catalogues are represented on the HRD. Two solar metallicity isochrones, corresponding to $1 \mathrm{Gyr}$ and $10 \mathrm{Gyr}$, are shown as reference. Since BTPH is a subsample extracted from TPH, all the cyan crosses representng the BTPH stars are superimposed on part of the blue reverse triangles representing the TPH stars.

and 0.63 dex, respectively. We further removed $F$ type stars, i.e stars with $T_{\text {eff }}>6300 \mathrm{~K}$, from the $\mathrm{SN}$ catalogue. This way we remained with 3713 stars (Reduced Solar Neighbourhood catalogue; RSN) belonging to the same spectral type range of planethosting stars. Among useful input parameters to compute stellar ages through our own algorithm, Casagrande et al. (2011) give only metallicity, which is inferred from Strömgren photometry; distance, according to the new reduction of the HIPPARCOS parallaxes (van Leeuwen 2007); and $V$ magnitude for each star. We complemented this information by cross-matching the entire Geneva-Copenhagen survey with the catalogue of cool late-type stars by Valenti \& Fischer (2005), which also provides precise spectroscopic measurements of surface gravity $\log g$ and projected rotational velocity $v \sin i$. This led to the Valenti Fischer Solar Neighbourhood catalogue (VF-SN catalogue), which contains 825 stars.

A brief overview of our custom-built catalogues used in the paper is given in Table 1. In Fig. 1 stars, belonging to our catalogues are represented on the HRD with two solar metallicity isochrones as reference.

\subsection{Isochrones}

To compute the ages of stars we used isochrones taken from Padova and Trieste Stellar Evolutionary Code (PARSEC, version 1.0) $)^{3}$ by Bressan et al. (2012). We queried isochrones identified by $\log t$ in the range between 6 and 10.1 ( $t$ in years) at steps of 0.05 dex. These isochrones include the pre-MS phase, so the given ages must be considered as starting from the birth of a star and not since the zero age main sequence (ZAMS). Specific details about the solar parameters adopted by the isochrones are already reported by Bonfanti et al. (2015). Here, we recall the relation between metallicity $Z$ and $[\mathrm{Fe} / \mathrm{H}]$, i.e.

$Z=10^{[\mathrm{Fe} / \mathrm{H}]-1.817}$

3 http://stev.oapd.inaf.it/cgi-bin/cmd 
Table 1. Overview of our custom-built catalogues.

\begin{tabular}{lcll}
\hline \hline Catalogue & \# Stars & $\log g$ source & Reference \\
\hline TPH: Transiting planet hosts & 61 & spectroscopy & SWEET-Cat \\
BTPH: Bright transiting planet hosts & 43 & spectroscopy & SWEET-Cat \\
SH: Spectroscopic hosts & 274 & spectroscopy & SWEET-Cat \\
SN: Solar neighbourhood & 7044 & not available & Casagrande et al. (2011) \\
RSN: Reduced solar neighbourhood & 3713 & not available & Casagrande et al. (2011) \\
VF-SN: Valenti Fischer solar neighbourhood & 825 & spectroscopy & Casagrande et al. (2011)+ \\
Kepler sample & & & Valenti \& Fischer (2005) \\
& 29 & asteroseismology & Silva Aguirre et al. (2015) \\
\hline
\end{tabular}

Notes. ${ }^{(a)}$ See Sect. 4.1.

\section{Age determination methods}

Computing the age of a field star through isochrones requires us to put the star on a suitable plane with its error bars. Traditionally, HRD is chosen, so $T_{\text {eff }}$ and $L$ are the reference quantities. Several catalogues in the literature already report $T_{\text {eff }}$ or $L$, but they were obtained by different authors through different processes and/or calibration techniques. For instance, $T_{\text {eff }}$ and $L$ are not likely to be consistent with the colourtemperature scale or the bolometric corrections (BCs) adopted by the isochrones. Therefore, we prefer to start from quantities coming from observations in a straightforward way, where possible. Our reference input quantities to compute stellar ages are $V$ magnitude, $B-V$ colour index, $[\mathrm{Fe} / \mathrm{H}]$ metallicity, spectroscopic $\log g$, and parallactic distance $d$, which can be substituted by the $\frac{a}{R_{\star}^{3}}$ parameter coming from transit, as better specified in Sect. 3.1.

\subsection{Isochrone placement: Preliminary considerations}

Starting from observational quantities, $T_{\text {eff }}$ can be inferred from colour index (e.g. $B-V$ ), while $L$ is determined thanks to the magnitude in a given band (say $V$ ), its corresponding bolometric correction $B C_{V}$ and the distance $d$ of the star recovered from parallax $\pi$. In the particular case where a star hosts a transiting planet, we are able to compute $L$, even if $d$ is not available. In fact, the ratio between the orbital period $P$ and the transit duration allows us to recover $a / R_{\star}$, where $a$ is the planet semimajor axis and $R_{\star}$ is the stellar radius (see e.g. Winn 2010). Rearranging Kepler III law in the manner shown by Sozzetti et al. (2007), the mean stellar density results to be

$\rho_{\star}=\frac{3 \pi}{G}\left(\frac{a}{R_{\star}}\right)^{3} \frac{1}{P^{2}}$

where $G$ is the universal gravitational constant. Combining the spectroscopic $\log g$ with $\rho_{\star}$, one can solve a system of two equations in the two variables $R_{\star}$ and $M_{\star}$. One obtains

$\left\{\begin{array}{l}\frac{R_{\star}}{R_{\odot}}=\frac{g}{g_{\odot}}\left(\frac{\rho_{\star}}{\rho_{\odot}}\right)^{-1} \\ \frac{M_{\star}}{M_{\odot}}=\left(\frac{g}{g_{\odot}}\right)^{3}\left(\frac{\rho_{\star}}{\rho_{\odot}}\right)^{-2} .\end{array}\right.$

Finally, the stellar luminosity $L$ is given by

$$
\frac{L}{L_{\odot}}=\left(\frac{R}{R_{\odot}}\right)^{2}\left(\frac{T_{\mathrm{eff}}}{T_{\mathrm{eff}, \odot}}\right)^{4} .
$$

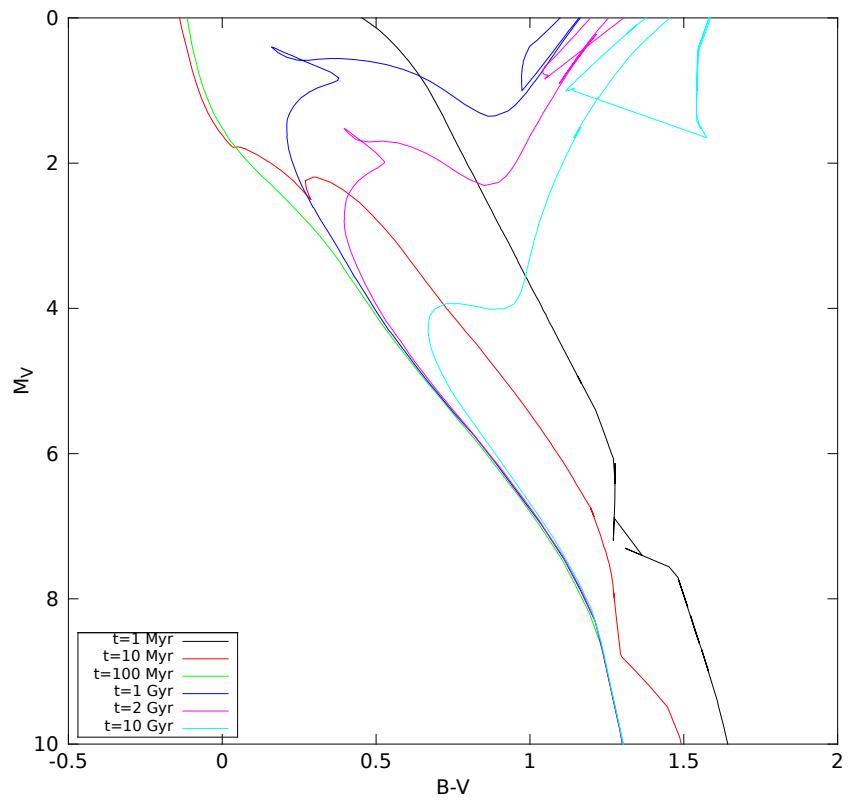

Fig. 2. Isochrones of solar metallicity: pre-MS isochrones are located on the right-hand side of the MS (in this region ages increase from right to left on the diagram) and they intersect older isochrones around the TO. The ages of MS isochrones increase from left to right on the diagram.

In Bonfanti et al. (2015) we have already pointed out that on the right-hand side of the main sequence (in the lower temperature region) or around the turn-off (TO) very old and very young isochrones are close and can even overlap. According to Fig. 2, the 1-Myr- and 10-Myr-isochrones interesect older isochrones in the TO region. The intersection points are representative of a degeneracy between pre-MS and MS isochrones on the CMD. In fact, the photometry alone cannot disentagle young and old ages, and other information is needed.

So far, the only exoplanet candidate orbiting around a preMS is PTFO 8-8695b, as reported by van Eyken et al. (2012) and then investigated by Barnes et al. (2013). Thus, we do not expect to find pre-MS stars among our samples of stars with planets. Anyway, our algorithm is built to compute ages of any kind of star and we decided to perform the activity checks that are described in the following. In this way, we do not put any a priori conditions on the evolutionary stage of the planet-hosting stars. Possible pre-MS interlopers in our planet-hosting stars samples would be very low-mass stars with long pre-MS lifetimes. Twenty-two stars out of the 335 SWP have masses lower than $0.8 M_{\odot}$. In principle, they could be pre-MS stars and the checks performed by our algorithm may help in recognizing them. 


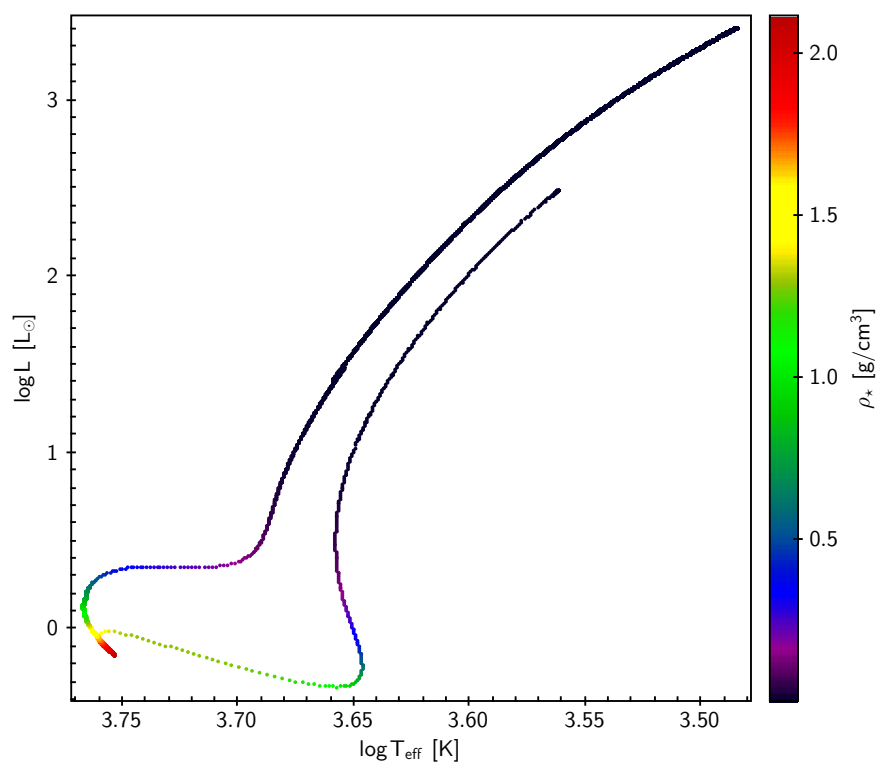

Fig. 3. Evolutionary track of a star with $M=1 M_{\odot}$ and $Z=Z_{\odot}$. Even if the oldest MS isochrones are close to pre-MS isochrones, however, $\rho_{\star}$ in the MS is sensibly higher with respect to the pre-MS phase. As a consequence, the mean stellar density enables us to discard unlikely age values according to $\rho_{\star}$.

In the case of late spectral type stars, such as those analysed in this paper, very young stars are chromospherically very active with respect to older stars and typically rotate faster, so we performed activity checks in terms of $\log R_{H K}^{\prime}$ and $v \sin i$, trying to remove the degeneracy between pre-MS and MS isochrones. We evaluated three age scales through three independent methods to decide on the ensemble of isochrones to be used in the following computation.

1. We considered the age-activity relation by Mamajek \& Hillenbrand (2008) and we set a conservative threshold of 0.2 dex corresponding to the typical difference between the highest and lowest peaks in activity and the average level for a solar-type star. Inserting $\log R_{H K}^{\prime}$ in the relation, we evaluated the corresponding age: $\tau_{H K}$ represents this age if it was younger than $500 \mathrm{Myr}$, otherwise $\tau_{H K}=500 \mathrm{Myr}$.

2. Meibom et al. (2015) proved that the gyrochronological relation by Barnes (2010) holds up to $2.5 \mathrm{Gyr}$, so we applied this relation employing $\frac{4}{\pi} v \sin i$ as the expected stellar rotational velocity to obtain the gyro age $\tau_{v}$. We set $\tau_{v}=2.5 \mathrm{Gyr}$, if the resulting gyro age was older than $2.5 \mathrm{Gyr}$.

3. There is other information that may suggest whether a star located under the TO on the right-hand side of the MS is very young or very old, and this is $\rho_{\star}$. Figure 3 shows the evolutionary track of a $1 M_{\odot}$ star with solar metallicity. Starting from the birth of a star, $\rho_{\star}$ increases in approaching the MS. After the TO, $\rho_{\star}$ clearly decreases so that post-MS stars have a mean stellar density similar to that of pre-MS stars. So, for $M_{V}>5$, corresponding to the luminosity of the TO of the oldest isochrone in the CMD, pre-MS isochrones differ from older isochrones in terms of $\rho_{\star}$. Among pre-MS ages, $\tau_{\rho}$ is the threshold age value such that for $t<\tau_{\rho}$ isochrones report mean stellar density $\rho<\rho_{\star}$.

The maximum value among $\tau_{H K}, \tau_{v}$ and $\tau_{\rho}$ represents the age up to which all the younger isochrones are discarded before the computation of stellar age.

\subsection{Isochrone placement: Implementation}

The isochrone placement technique enables the determination of the ages of field stars, as well as all the other stellar parameters, such as $T_{\text {eff }}, L, \log g, M_{\star}, R_{\star}$, according to stellar evolutionary models. This technique was already described in Bonfanti et al. (2015), but several improvements have been made, such as the new kind of activity checks described above and the possibility of computing the age without any input distance $d$, if we have stellar density measurements. We also solved some problems linked to numerical stability convergence for which the previous algorithm sometimes gave fictitious young ages. In fact, in the previous version of the algorithm some input data were loaded in single precision, instead of double precision. Sometimes, it could happen that single precision were not sufficient to perform the correct computation of stellar parameters. Moreover, we make this new version more flexible, since it also enables to use input asteroseismic global parameters or only spectroscopic parameters if photometry is not available. Here, we briefly summarize the key aspects.

To make as few assumptions as possible and to start from input data directly obtained from observations, our algorithm requires

\section{- visual magnitude $V$; \\ - colour index $B-V$; \\ - metallicity $[\mathrm{Fe} / \mathrm{H}]$; \\ - spectroscopic $\log g$;}

and the distance $d$ or $a / R_{\star}$. If $d$ is available, it is possible to infer $T_{\text {eff }}$ from $B-V$ and $L$ from the absolute magnitude $M_{V}$ via interpolation in the isochrone grid. Then $R_{\star}$ is known thanks to Stefan-Boltzmann law (Eq. (4)) and finally $M_{\star}$ can be computed by combining $R_{\star}$ with $\log g$. If, instead, $d$ is not available, which can occur for some TPH, first we compute $\rho_{\star}$ through (2) and then we recover $M_{\star}$ and $R_{\star}$ via relations (3). After that, we obtain the correspondence between $B-V$ and $T_{\text {eff }}$ through interpolation in the isochrone grid. Finally, we compute $L$ from $T_{\text {eff }}$ with (4). Once all the input parameters are available, it is possible to derive stellar properties according to Padova theoretical models by properly weighting each isochrone in the manner already described in Bonfanti et al. (2015). With the new version of the algorithm, we improved the weighting scheme to take the evolutionary speed of a star into account. In fact, the probability that a star is a given age does not only depend on the simple distance between the star and the given isochrone in the HRD, but it is also influenced by the time spent by a star in a given evolutionary stage. For instance, pre-MS evolution is quicker than the MS one. This means that a star rapidly changes its properties during the first tenths of Myr of its life, instead, it remains in the MS for Gyrs with parameters variations detectable on longer timescales. As a consequence, given a star on the HRD, located at the same distance with respect to a pre-MS and a MS isochrone, the probability of dealing with a MS star is higher. To quantify this aspect, we considered the theoretical stellar evolutionary track characterized by the same input metallicity and mass of the star, and we evaluated its evolutionary speed by

$v_{\mathrm{evo}}=\sqrt{\left(\frac{\log L_{2}-\log L_{1}}{t_{2}-t_{1}}\right)^{2}+\left(\frac{\log T_{\mathrm{eff}, 2}-\log T_{\mathrm{eff}, 1}}{t_{2}-t_{1}}\right)^{2}}$

where $\left(\log T_{\text {eff, }, 1}, \log L_{1}\right)$ is the point on the track, that is nearer to the star, while $\left(\log T_{\text {eff, }, 2}, \log L_{2}\right)$ is the point that occurs later in time on the track, and $t_{1}$ and $t_{2}$ are the epochs reported by the 
track. The greater $v_{\text {evo }}$, the less is the probability to find a star in such an evolutionary stage. We normalized $v_{\text {evo }}$, with respect to a reference speed value $v_{\text {ref }}$ for a given track, that is the lowest speed registered on the entire track. In this way, the evolutionary speed can be easily interpreted as a multiple of a reference speed with which a star goes along its track and the contribution to be added to the weight is unitless, like the others. So the weight $p_{i}$ to be attributed to the $i$ th isochrone results to be

$$
\begin{aligned}
p_{i}= & {\left[\left(\frac{\log L-\log L_{i}}{\Delta \log L}\right)^{2}+\left(\frac{\log T_{\mathrm{eff}}-\log T_{\mathrm{eff}, i}}{\Delta \log T_{\mathrm{eff}}}\right)^{2}\right.} \\
& \left.+\left(\frac{M_{\star}-M_{\star, i}}{\Delta M_{\star}}\right)^{2}+\left(\frac{\log g-\log g_{i}}{\Delta \log g}\right)^{2}+\log ^{2}\left(\frac{v_{\mathrm{ref}}}{v_{\mathrm{evo}}}\right)\right]^{-1} .
\end{aligned}
$$

Sometimes photometry is not available and only spectroscopic analyses have been carried out. We caution that the given spectroscopic input temperature has been inevitably subjected to a calibration process, which can bring biases. Anyway, to compute ages in such cases we can use spectroscopic $[\mathrm{Fe} / \mathrm{H}], T_{\text {eff }}$ and $\log g$. In this case, the algorithm works in the $\log g-\log T_{\text {eff }}$ plane following the same prescriptions as in the HRD. This time the weight is simply given by

$p_{i}=\left[\left(\frac{\log g-\log g_{i}}{\Delta \log g}\right)^{2}+\left(\frac{\log T_{\text {eff }}-\log T_{\text {eff }, i}}{\Delta \log T_{\text {eff }}}\right)^{2}+\log ^{2}\left(\frac{v_{\text {ref }}}{v_{\text {evo }}}\right)\right]^{-1}$

where the evolutionary speed of the star $v_{\text {evo }}$ and its reference value $v_{\text {ref }}$ are evaluated in the $\log g-\log T_{\text {eff }}$ plane instead of the HRD.

If global asteroseismological indexes, i.e. $\Delta v$ and $v_{\max }$, are available, $\log g$ and $\rho_{\star}$ may also be computed by inverting the following scaling relations:

$\Delta v=\sqrt{\frac{M_{\star}}{M_{\odot}}\left(\frac{R_{\star}}{R_{\odot}}\right)^{-3}} \Delta v_{\odot}$

$v_{\max }=\frac{g}{g_{\odot}}\left(\frac{T_{\text {eff }}}{T_{\text {eff }, \odot}}\right)^{-\frac{1}{2}} v_{\max , \odot}$

$\Delta v_{\odot}=135.1 \mu \mathrm{Hz}$ and $v_{\max , \odot}=3090 \mu \mathrm{Hz}$ as reported by Chaplin et al. (2014). Knowledge of both $\log g$ and $\rho_{\star}$ enables us to compute $M_{\star}$ and $R_{\star}$. Given that $T_{\text {eff }}$ is available, $L$ may also be recovered using (4). Even if an accurate asteroseismological analysis based on the study of individual frequency enables precise determination of the stellar evolutionary stage, however, combining information from global asteroseismic parameters and from spectroscopy gives a complete set of input data useful for our isochrone placement. Once the star is located on the HRD, it is then possible to compute its age and its parameters according to Padova evolutionary models. Our algorithm takes element diffusion into account.

If known, stellar multiplicity has been pointed out through a flag at the column Bin of Table A.1. In these cases, the literature already reports data referred to the specific star we analysed. We caution that if some unresolved binaries were present in our samples, such stars would appear more luminous than they are. If located in the MS region, they would erroneously be judged as older.

Another critical point deals with reddening. In the case of SWP, we do not deeply check whether the different sources give photometry de-reddened or not because neither SWEET-Cat nor the Exoplanets Data Explorer report any reddening information. We explicitely account for reddening in the case of those TPH listed in Maxted et al. (2011), who report the colour excess $E(B-V)$. Anyway, by a posteriori catalogue cross-matching, we were able to recover $E(B-V)$ index for 154 stars out of the 335 SWP, and we found that more than $80 \%$ of them has $E(B-V)=0$. Similarly, $\sim 90 \%$ of the stars belonging to the VF-SN catalogue have $E(B-V)=0$. Considering also that the analysed stars are essentially inside the Local Bubble, whose extension varies between $\sim 80$ and 200 pc from the Sun (Sfeir et al. 1999), we expect that the effect of reddening does not significantly impact our resulting statistics.

\section{Results}

\subsection{Test of the algorithm}

Silva Aguirre et al. (2015) analysed a sample of Kepler exoplanet host stars (Kepler sample from here on). They performed a complete asteroseismological analysis of the individual oscillation frequencies, recovered thanks to the high signal-to-noise ratio of their observations. Among other fundamental properties, they derived the ages of their sample of stars, claiming a median error of $14 \%$. As a result of high reliability attributed to a complete asteroseismological analysis, comparing the results given by our isochrone placement with those reported by Silva Aguirre et al. (2015) represents a good validation test for our algorithm. The authors observe that the majority of these stars are older than the Sun because of selection effects. In particular, stellar pulsations characterized by high signal-to-noise ratio are preferentially detected in F-type stars (ages 2-3 Gyr) and in old G-type stars (ages $\sim 6 \mathrm{Gyr}$ ). Thus, aim of this section is to test the accuracy of our algorithm, without comparing the evolutionary stage of the Kepler sample with other stars.

We analysed 29 over 33 stars of the Kepler sample, for which both $\Delta v$ and $v_{\max }$ were available. We have just considered the global asteroseismic parameters, deriving input $\log g$ and $\rho_{\star}$ by inverting (8) and (9). Spectroscopic $[\mathrm{Fe} / \mathrm{H}]$ and $T_{\text {eff }}$ were reported by Silva Aguirre et al. (2015). If available, $v \sin i$ was employed to perform checks as described in point 2 in Sect. 3.1.

Our age determination is in good agreement with the analysis of Silva Aguirre et al. (2015), as shown in Fig. 4. The linear correlation coefficient $r=0.95$ and the reduced $\chi^{2} / 26=1.5$ confirm that a linear least-squares regression well describe the data scatter and is consistent with the extension of our error bars. The least-squares line represented in green (thicker line) shows that our method slightly overestimate the age in the domain of the oldest stars.

\subsection{The ages of the exoplanet hosts}

The BTPH catalogue is a subset of the TPH catalogue. In Fig. 5, we superimposed the age distribution of the 43 stars belonging to the BTPH catalogue (grey bars) to the age distribution of the all TPH (blue bars). The medians of the distributions are $4.2 \mathrm{Gyr}$ and $\sim 5$ Gyr for the BTPH and for the TPH, respectively. One of the three stars younger than $1 \mathrm{Gyr}$, namely WASP-18 $(t=$ $0.9 \pm 0.2 \mathrm{Gyr}$ ), appears too blue for its metallicity, so we investigated the input parameters of this star. Southworth et al. (2009) analysed the properties of WASP-18, adopting $V=9.30$ and $B-V=0.44$, instead of $V=9.273$ and $B-V=0.484$, which are the values we used. In addition they started from $[\mathrm{Fe} / \mathrm{H}]=0$, as reported by Hellier et al. (2009), which sensibly differs from $[\mathrm{Fe} / \mathrm{H}]=0.19$, which we took from SWEET-Cat. With the input 


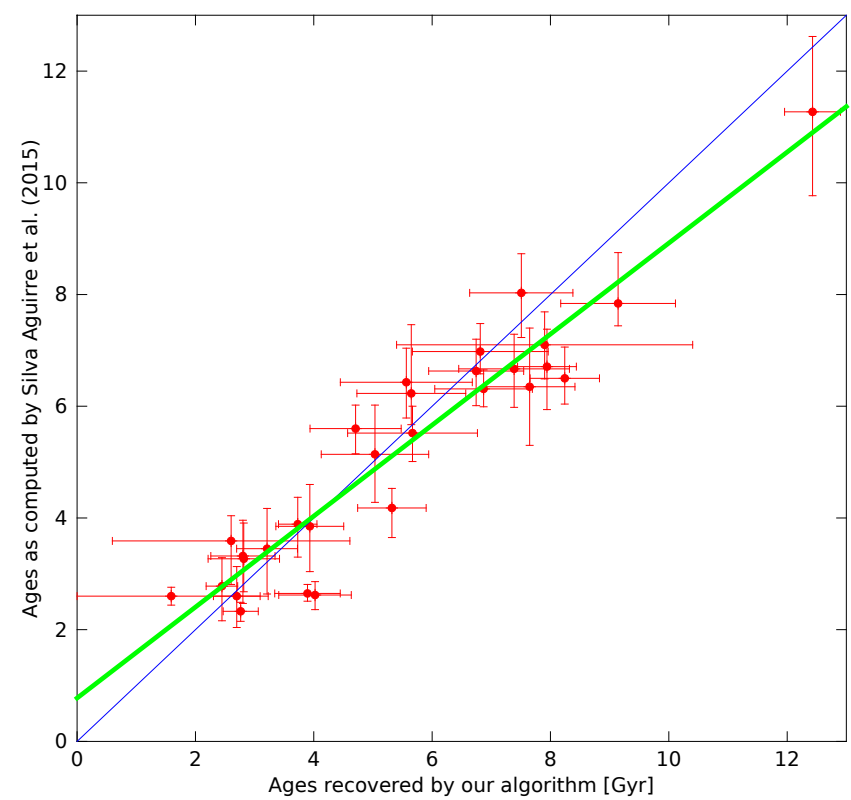

Fig. 4. Ages of the Kepler sample. The least-squares line that regress the data is represented with a thick green line, while the thin blue line is the bisector representing the exact correspondence between the data.

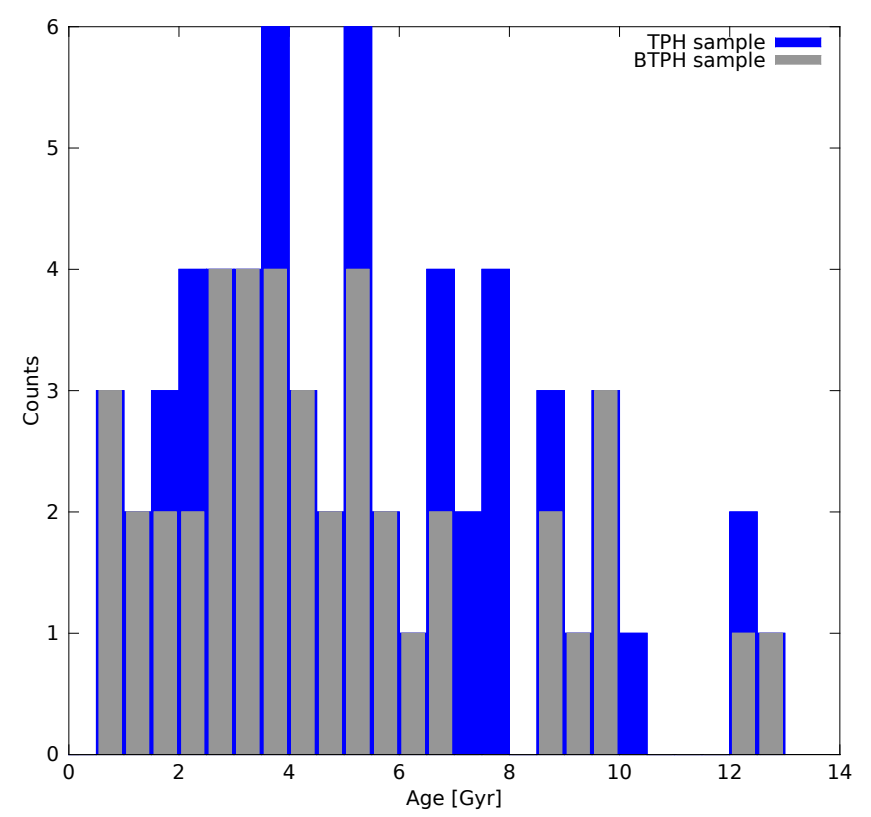

Fig. 5. Grey bars represent age distribution of the 43 stars belonging to the BTPH catalogue. The superset given by TPH catalogue is represented in the background through blue bars.

parameters used by Southworth et al. (2009), WASP-18 is again located on the bluer side out of the MS, and our isochrone placement gives $t=0.2_{-0.2}^{+0.3}$ Gyr. Southworth et al. (2009) considered different sets of evolutionary models and they concluded that WASP-18 is age between 0 and 2 Gyr. This is consistent with both of our determinations, but since the different sets of input parameters do not fully agree with Padova theoretical models, we caution that the age estimation is not necessarily reliable. Further photometric investigations or a re-determination of its metallicity are required to correct the inconsistency between the position of the star on the HRD and the theoretical isochrones.

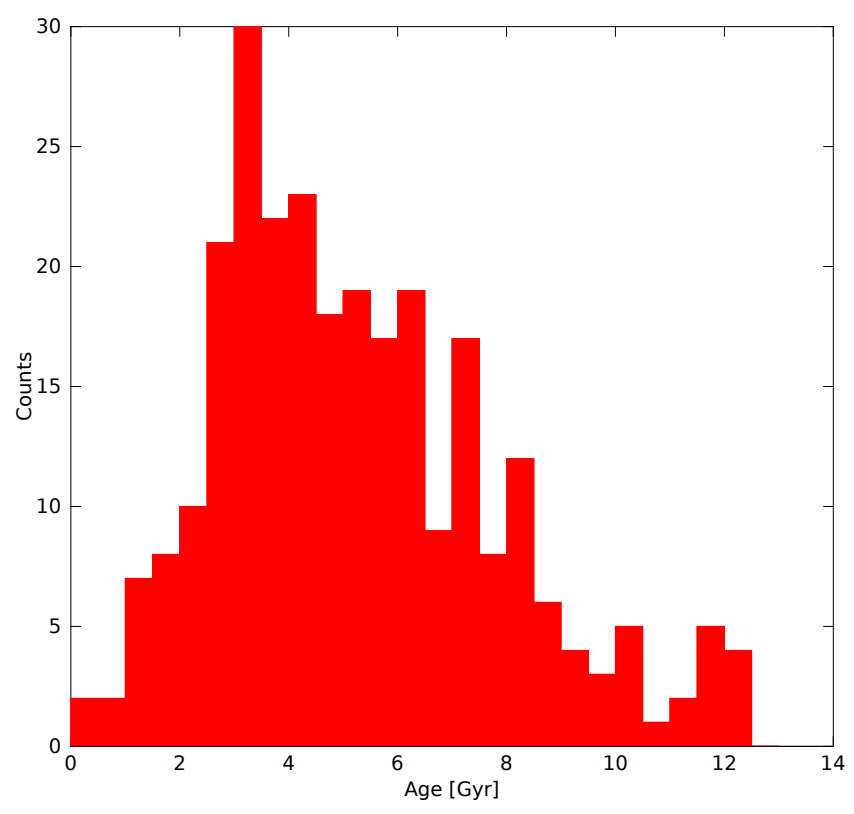

Fig. 6. Age distribution of the 274 stars belonging to the $\mathrm{SH}$ catalogue. The median of the distribution is $\sim 4.8 \mathrm{Gyr}$, which is very close to the age of the Sun.

As a term of comparison, we computed the ages of stars taken from $\mathrm{SH}$ catalogue. The consequent age distribution is represented in Fig. 6. This age distribution peaks in the [3, 3.5) Gyr bin and its median is $\sim 4.8 \mathrm{Gyr}$, which is very close to the solar age value. The age distributions in Figs. 5 and 6 are consistent. Differences may arise because of the paucity of the TPH, but, in any case, no significant bias emerges in the comparison between the samples. Actually, we performed a KolmogorovSmirnov test (KS test) to investigate whether TPH and BTPH come from the same distribution, which characterizes the larger $\mathrm{SH}$ sample. The high p-values (0.5 for the TPH-SH comparison and 0.3 for the BTPH-SH comparison) suggest that we should not reject the null hypothesis based on which the samples come from the same distribution. This emphasizes that even if photometric and spectroscopic targets could be chosen according to different criteria, the confirmation of a candidate exoplanet requires the application of both the transit and radial velocity method. Therefore, similar biases are expected in the two different samples.

All the parameters of the planet-hosting stars derived according to Padova isochrones are listed in Table A.1.

\subsection{Age comparison with the stars of the solar neighbourhood}

Our second step is to investigate whether exoplanet hosts are peculiar with respect to field stars not harbouring planets. The exoplanet hosts known so far are late spectral type stars located in the solar neighbourhood: $\sim 90 \%$ of the planet-hosting stars we analysed are closer than $200 \mathrm{pc}$. The stars contained in the SN catalogue represent an interesting comparison test because they represent a numerous sample of late spectral type MS-stars occupying almost the same volume as the exoplanet hosts. The age values reported by Casagrande et al. (2011, C11 from here on) are computed following their own method, so we checked whether their results were consistent following the VF-SN subsample, which contains all the input parameters needed by our algorithm. We managed to obtain the age for 818 stars. We recall 
A. Bonfanti et al.: Age consistency between exoplanet hosts and field stars

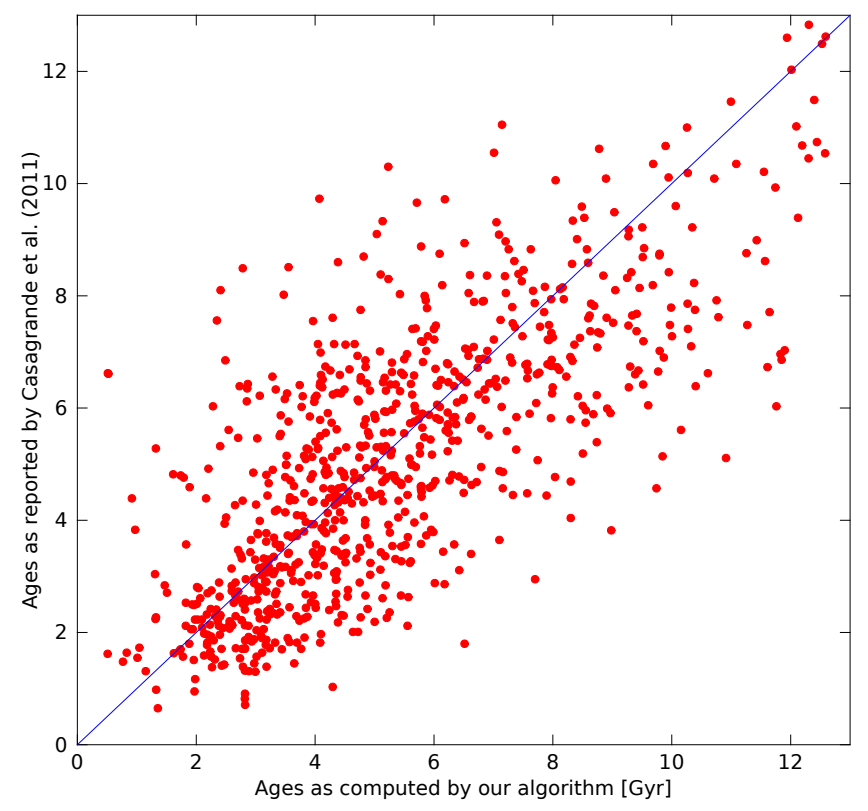

Fig. 7. Age comparison between Casagrande estimation and ours (818 stars). The dispersion between the two measurements can be quantified through the linear correlation coefficient, that is $\sim 0.75$.

that the cut in spectral type is imposed by both $\mathrm{C} 11$ and Valenti \& Fischer (2005) limits, and includes basically F, G and K stars. The plot of the expectation age reported by $\mathrm{C} 11$ versus our age values is shown in Fig. 7. The scatter of points around the bisector is expected given the high age uncertainties, and, in any case, good statistical agreement characterizes the two determinations. The median age for $\mathrm{C} 11$ values is $\sim 4.9 \mathrm{Gyr}$, which is very similar to our median age ( $\sim 4.8 \mathrm{Gyr})$ for the common sample. This agreement between the two age determinations suggests that any comparison between $\mathrm{C} 11$ ages and ours is consistent. In addition, considering the median age value coming from the VF-SN sample, it is not surprising that the age is similar to the ages found for the samples of stars with planets analysed above. In fact, we obtained the VF-SN catalogue by cross-matching the SN sample with the catalogue of stars reported by Valenti $\&$ Fischer (2005). The authors performed high-precision spectroscopy on stars taken from Keck, Lick and AAT planet search programme, thus, their stars present the typical selection effects characterizing stars with planets. Actually the median value we obtained for the VF-SN sample is the same as the SH value.

As C11 age values suggest, the age distribution of all the 7044 stars belonging to the SN catalogue peaks in the [1.5, 2) Gyr bin with a median age value of $\sim 2.6$ Gyr. This raw analysis may suggest that field stars are globally younger than planethosting stars. Instead, this comparison hides a bias, in fact, the $\mathrm{SN}$ catalogue contains a huge number of hot F-type stars with respect to planet-hosting stars, as shown in Fig. 8 (stars with $\log T_{\text {eff }} \gtrsim 3.8$ ). The earlier the spectral type, the faster the evolution of a star, thus, F-type stars are expected to be statistically younger than later spectral type stars.

Analysing Fig. 8, all the stars with $T_{\text {eff }}>6300$ have been removed from the SN catalogue, remaining with 3713 stars (RSN catalogue). In this way, the comparison between the solar neighbourhood stars and the stars with planets is homogeneous in spectral type. The result is given by the age distributions in Fig. 9. They are almost consistent (KS p-value is 0.2): SN age distribution is less peaked, but they both have $4.8 \mathrm{Gyr}$ as median value.

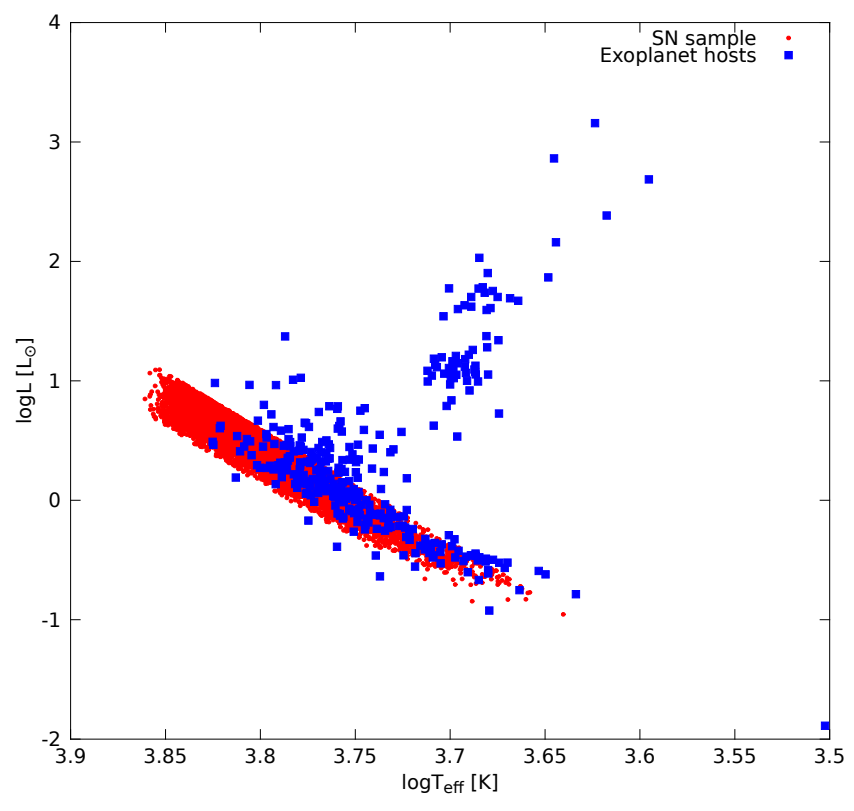

Fig. 8. SN sample on the HRD (red dots). The very straight boundaries of the SN sample is simply a consequence of our identification of the MS through a strip as described in Sect. 2.2. All the planet-hosting stars we analysed are superimposed (blue squares).

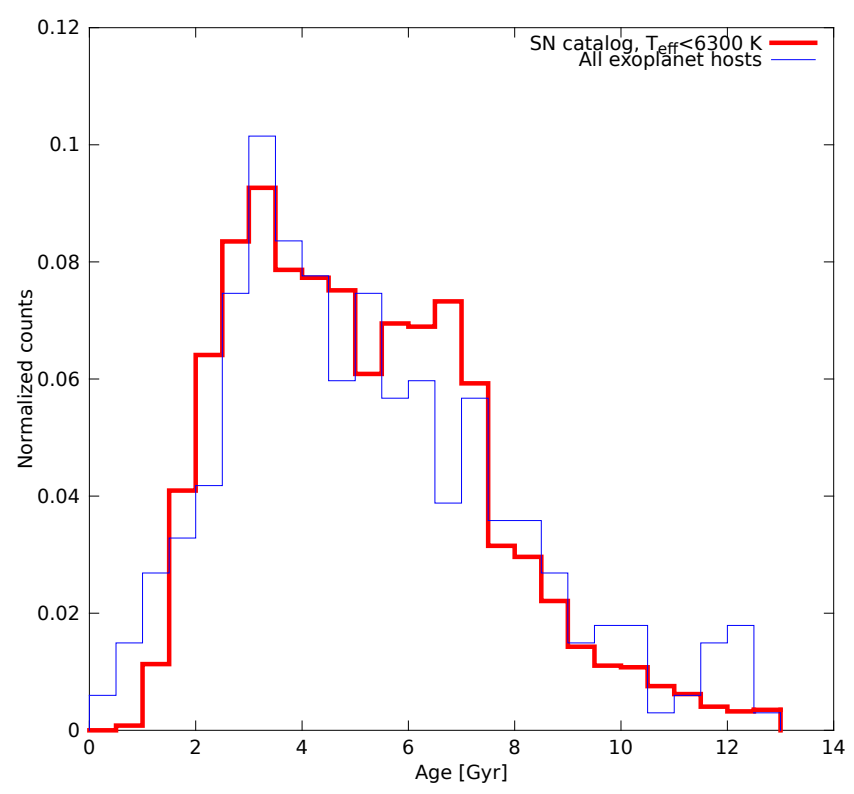

Fig. 9. Age distribution of the MS stars colder than $6300 \mathrm{~K}$ belonging to the RSN catalogue (red stairs, 3713 stars) compared with the age distribution of all the planet-hosting stars we analysed (blue stairs, 335 stars).

Two considerations should be added.

1. The median age value of the stars with planets is very similar to the solar age value, but very different $(\sim 1 \mathrm{Gyr})$ from the histogram peak because of the extended tail towards old ages. According to their metallicities, there is no evidence that planet-hosting stars with $t \gtrsim 9$ Gyr have population II properties. If we exclude this contamination, we may argue whether such a skew distribution may be due to a real distribution reflecting a prolonged star formation history (RochaPinto et al. 2000) or to an asymmetric propagation of errors.

2. Assuming a solar age of $4.6 \mathrm{Gyr}$ (Chaussidon 2007), an age of $t=4.6-4.8 \mathrm{Gyr}$ appears older than that currently assumed 
for most of the thin disc population, where planet-hosting stars and the RSN sample are located. In fact, as summarized by Allende Prieto (2010), Reddy et al. (2006) say that thin disc stars span a range between 1 and 9 Gyr, with the majority of them younger than 5 Gyr. Holmberg et al. (2009) and Haywood (2008) set an older upper limit for the thin disc ages, however they both agree that most of these stars are younger than 4-5 Gyr. Rocha-Pinto et al. (2000), using a different approach based on the stellar activity as age indicator, found three different peaks in the local star formation history, with the highest at very young ages below 1 Gyr. We caution that we limited our sample primarily to G-type stars. The other point is that we are sampling a very limited inter-arms volume (essentially $<200 \mathrm{pc}$ ), as most of the recent studies based on single star age measurements. The literature does not present detailed studies of the ages of single disc stars far away from the solar neighbourhood. Thus, this lack of information does not allow us to perform a complete comparison between the evolutionary properties of our samples and those of the entire galactic disc. The extension of the stellar analysis to a distance larger than $200 \mathrm{pc}$ would include younger active star-forming regions, such as the Orion Nebula or Taurus-Auriga complex. As a consequence, in deeper surveys we expect to include significantly younger stars.

\section{Conclusions}

We analysed a sample of 61 transiting-planet hosts to compute their ages and their peculiar parameters according to Padova isochrones. A priori, one could say that our particular sample is expected not to be affected by some typical biases that characterize those planet-hosting stars coming from radial velocity surveys. Spectroscopic targets are often deliberately chosen to be slow rotators and typically inactive. Instead, very high-precision photometry only requires bright stars in the solar neighbourhood for an adequate signal-to-noise ratio. Actually, once a possible transiting planet has been detected, the confirmation process involves spectroscopic analysis. Therefore, similar biases are expected in both spectroscopic and photometric surveys.

We found that the median age of our TPH sample is $5 \mathrm{Gyr}$. The subsample of TPH brighter than $V=12$ yields a median age of $\sim 4.2$ Gyr. This slightly lower value is expected since brighter stars are on average younger. In order to comment the age distribution of TPH, we also considered 274 planet-hosting stars, whose planets have been detected though radial velocity method. Their age distribution peaks in the $[3,3.5)$ Gyr bin and it is synthesized by a median value of $\sim 4.8 \mathrm{Gyr}$. These three samples of stars are consistent from an evolutionary point of view. Slight differences are due to the paucity of stars belonging to the TPH catalogue and, in fact, a KS test does not suggest that TPH and $\mathrm{SH}$ come from a different distribution. Thus, spectroscopic and photometric targets are characterized by almost the same selection effects, and these biases bring the median of their age distribution around the solar age value.

In the second part we checked whether planet-hosting stars have peculiar ages with respect to field stars without planets of the solar neighbourhood. In case of a homogeneous comparison in terms of spectral type, solar neighborhood stars belonging to the RSN catalog have an age distribution very similar to that deriving from the all exoplanet hosts considered in this paper and the median age is $\sim 4.8$ in both cases. With its age of $4.567 \mathrm{Gyr}$ (as reported by Chaussidon 2007), the Sun appears not to be a peculiar star, if compared with both the planet-hosting stars and the SN stars, whose spectral types span essentially from late-F to early K. However, it looks that we are sampling a limited interarms region, possibly older than the average thin disc population.

Acknowledgements. We thank the anonymous referee for the interesting and fruitful comments that improved our paper. A.B. and S.O. acknowledge "contratto ASI-INAF n.2015-019-R0 del 29 luglio 2015" and support from INAF through the "Progetti Premiali" funding scheme of the Italian Ministry of Education, University, and Research. S.O. acknowledge financial support from University of Padova, as well. V.N. acknowledges support from INAF-OAPd through the grant "Studio preparatorio per le osservazioni della missione ESA/CHEOPS" (\#42/2013). This research has made use of the Exoplanet Orbit Database and the Exoplanet Data Explorer at exoplanets.org. Moreover it has made use of SWEET-Cat: a catalog of stellar parameters for stars with planets at astro.up.pt (Centro de Astrofisica da Universidade do Porto).

\section{References}

Allende Prieto, C. 2010, in IAU Symp. 265, eds. K. Cunha, M. Spite, \& B. Barbuy, 304

Barker, A. J. \& Ogilvie, G. I. 2009, MNRAS, 395, 2268

Barnes, S. A. 2010, ApJ, 722, 222

Barnes, S. A. \& Kim, Y.-C. 2010, ApJ, 721, 675

Barnes, J. W., van Eyken, J. C., Jackson, B. K., Ciardi, D. R., \& Fortney, J. J. 2013, ApJ, 774, 53

Bonfanti, A., Ortolani, S., Piotto, G., \& Nascimbeni, V. 2015, A\&A, 575, A18

Bressan, A., Marigo, P., Girardi, L., et al. 2012, MNRAS, 427, 127

Broeg, C., Fortier, A., Ehrenreich, D., et al. 2013, in EPJ Web Conf., 47, 3005

Casagrande, L., Schönrich, R., Asplund, M., et al. 2011, A\&A, 530, A138

Chaplin, W. J., Basu, S., Huber, D., et al. 2014, ApJS, 210, 1

Chaussidon, M. 2007, Formation of the Solar system: a chronology based on meteorites, eds. M. Gargaud, H. Martin, \& P. Claeys (Berlin: Springer-Verlag), 45

Debes, J. H. \& Jackson, B. 2010, ApJ, 723, 1703

Handler, G. 2013, Asteroseismology (Oswalt, T. D. and Barstow, M. A.), 207 Haywood, M. 2008, MNRAS, 388, 1175

Hellier, C., Anderson, D. R., Collier Cameron, A., et al. 2009, Nature, 460, 1098

Holmberg, J., Nordström, B., \& Andersen, J. 2009, A\&A, 501, 941

Kasting, J. F. \& Catling, D. 2003, ARA\&A, 41, 429

Kjeldsen, H. \& Bedding, T. R. 1995, A\&A, 293, 87

Maeder, A. 1999, A\&A, 347, 185

Mamajek, E. E. \& Hillenbrand, L. A. 2008, ApJ, 687, 1264

Maxted, P. F. L., Koen, C., \& Smalley, B. 2011, MNRAS, 418, 1039

Meibom, S., Barnes, S. A., Platais, I., et al. 2015, Nature, 517, 589

Pätzold, M., Carone, L., \& Rauer, H. 2004, A\&A, 427, 1075

Rauer, H., Catala, C., Aerts, C., et al. 2014, Exper. Astron., 38, 249

Reddy, B. E., Lambert, D. L., \& Allende Prieto, C. 2006, MNRAS, 367, 1329

Rocha-Pinto, H. J., Scalo, J., Maciel, W. J., \& Flynn, C. 2000, A\&A, 358, 869

Santos, N. C., Sousa, S. G., Mortier, A., et al. 2013, A\&A, 556, A150

Sfeir, D. M., Lallement, R., Crifo, F., \& Welsh, B. Y. 1999, A\&A, 346, 785

Silva Aguirre, V., Davies, G. R., Basu, S., et al. 2015, MNRAS, 452, 2127

Soderblom, D. R. 2010, ARA\&A, 48, 581

Southworth, J., Hinse, T. C., Dominik, M., et al. 2009, ApJ, 707, 167

Sozzetti, A., Torres, G., Charbonneau, D., et al. 2007, ApJ, 664, 1190

Valenti, J. A. \& Fischer, D. A. 2005, ApJS, 159, 141

van Eyken, J. C., Ciardi, D. R., von Braun, K., et al. 2012, ApJ, 755, 42

van Leeuwen, F. 2007, A\&A, 474, 653

von Zeipel, H. 1924, MNRAS, 84, 665

Winn, J. N. 2010, ArXiv e-prints [arXiv: 1001 . 2010] 
A. Bonfanti et al.: Age consistency between exoplanet hosts and field stars

Appendix A: Additional table

Table A.1. Planet-hosting stars parameters determined through Padova isochrones.

\begin{tabular}{|c|c|c|c|c|c|c|c|c|c|c|c|c|c|c|}
\hline Star & $\begin{array}{r}t \\
(\mathrm{Gyr})\end{array}$ & $\begin{array}{r}\Delta t \\
(\mathrm{Gyr})\end{array}$ & $\begin{array}{l}T_{\text {eff }} \\
(\mathrm{K})\end{array}$ & $\begin{array}{r}\Delta T_{\text {eff }} \\
(\mathrm{K}) \\
\end{array}$ & $\begin{array}{r}L \\
\left(L_{\odot}\right)\end{array}$ & $\begin{array}{r}\Delta L \\
\left(L_{\odot}\right)\end{array}$ & $\begin{array}{r}M \\
\left(M_{\odot}\right) \\
\end{array}$ & $\begin{array}{r}\Delta M \\
\left(M_{\odot}\right)\end{array}$ & $\begin{array}{r}\log g \\
\left(\mathrm{~cm} / \mathrm{s}^{2}\right)\end{array}$ & $\begin{array}{r}\Delta \log g \\
\left(\mathrm{~cm} / \mathrm{s}^{2}\right)\end{array}$ & $\begin{array}{r}R \\
\left(R_{\odot}\right)\end{array}$ & $\begin{array}{r}\Delta R \\
\left(R_{\odot}\right)\end{array}$ & Bin & $\operatorname{Tr}$ \\
\hline $11 \mathrm{Com}$ & 3.0 & 0.3 & 4741 & 12 & 109 & 1 & 1.37 & 0.04 & 2.19 & 0.02 & 15.5 & 0.2 & 1 & 0 \\
\hline $11 \mathrm{UMi}$ & 4.1 & 1.0 & 4140 & 28 & 243 & 6 & 1.4 & 0.1 & 1.6 & 0.04 & 30.4 & 0.8 & 0 & 0 \\
\hline 14 And & 5.6 & 3.0 & 4775 & 49 & 56 & 1 & 1.2 & 0.2 & 2.4 & 0.1 & 11.0 & 0.3 & 0 & 0 \\
\hline $14 \mathrm{Her}$ & 4.6 & 1.5 & 5313 & 18 & 0.61 & 0.01 & 0.97 & 0.01 & 4.48 & 0.02 & 0.93 & 0.01 & 0 & 0 \\
\hline 16 Cyg B & 5.2 & 1.0 & 5837 & 32 & 1.26 & 0.01 & 1.04 & 0.02 & 4.37 & 0.02 & 1.1 & 0.02 & 1 & 0 \\
\hline $18 \mathrm{Del}$ & 1.0 & 0.1 & 5037 & 57 & 35 & 1 & 2.1 & 0.1 & 2.98 & 0.04 & 7.8 & 0.3 & 0 & 0 \\
\hline $24 \mathrm{Sex}$ & 2.9 & 0.2 & 4917 & 10 & 16.4 & 0.1 & 1.49 & 0.02 & 3.11 & 0.01 & 5.6 & 0.04 & 0 & 0 \\
\hline 30 Ari B & 0.4 & 0.4 & 6313 & 24 & 1.98 & 0.03 & 1.25 & 0.02 & 4.38 & 0.01 & 1.18 & 0.02 & 1 & 0 \\
\hline 42 Dra & 6.5 & 1.7 & 4414 & 40 & 145 & 3 & 1.1 & 0.1 & 1.8 & 0.1 & 20.6 & 0.6 & 0 & 0 \\
\hline $51 \mathrm{Peg}$ & 3.8 & 1.1 & 5857 & 39 & 1.34 & 0.03 & 1.09 & 0.02 & 4.37 & 0.02 & 1.13 & 0.03 & 0 & 0 \\
\hline $55 \mathrm{Cnc}$ & 5.1 & 2.7 & 5310 & 32 & 0.59 & 0.01 & 0.95 & 0.02 & 4.49 & 0.03 & 0.91 & 0.02 & 1 & 1 \\
\hline 6 Lyn & 3.1 & 0.4 & 4973 & 28 & 14.8 & 0.1 & 1.4 & 0.1 & 3.15 & 0.03 & 5.2 & 0.1 & 0 & 0 \\
\hline 61 Vir & 5.2 & 2.1 & 5651 & 36 & 0.81 & 0.01 & 0.96 & 0.02 & 4.46 & 0.03 & 0.94 & 0.02 & 0 & 0 \\
\hline $7 \mathrm{CMa}$ & 4.9 & 1.0 & 4782 & 39 & 11.3 & 0.2 & 1.3 & 0.1 & 3.17 & 0.04 & 4.9 & 0.1 & 0 & 0 \\
\hline 70 Vir & 8.1 & 0.4 & 5560 & 31 & 3.0 & 0.1 & 1.07 & 0.01 & 3.92 & 0.02 & 1.9 & 0.1 & 0 & 0 \\
\hline 75 Cet & 3.3 & 0.7 & 4742 & 14 & 53.7 & 0.3 & 1.4 & 0.1 & 2.52 & 0.03 & 10.9 & 0.1 & 0 & 0 \\
\hline $81 \mathrm{Cet}$ & 3.2 & 0.5 & 4799 & 24 & 59.8 & 0.4 & 1.4 & 0.1 & 2.49 & 0.03 & 11.2 & 0.2 & 0 & 0 \\
\hline $91 \mathrm{Aqr}$ & 8.1 & 2.8 & 4656 & 26 & 51 & 1 & 1.1 & 0.2 & 2.4 & 0.1 & 11.0 & 0.2 & 0 & 0 \\
\hline $\mathrm{BD}+144559$ & 6.9 & 4.2 & 4948 & 25 & 0.32 & 0.01 & 0.82 & 0.02 & 4.57 & 0.03 & 0.78 & 0.02 & 0 & 0 \\
\hline BD +20 2457 & 10.5 & 1.7 & 4504 & 5 & 172 & 12 & 0.89 & 0.05 & 1.71 & 0.01 & 21.6 & 0.8 & 0 & 0 \\
\hline CoRoT-18 & 7.5 & 4.5 & 5444 & 38 & 0.58 & 0.04 & 0.87 & 0.03 & 4.5 & 0.05 & 0.86 & 0.04 & 0 & 1 \\
\hline CoRoT-19 & 5.1 & 0.8 & 6133 & 58 & 3.0 & 0.3 & 1.2 & 0.1 & 4.12 & 0.01 & 1.5 & 0.1 & 0 & 1 \\
\hline CoRoT-7 & 4.5 & 4.3 & 5357 & 22 & 0.52 & 0.05 & 0.89 & 0.03 & 4.54 & 0.05 & 0.84 & 0.05 & 0 & 1 \\
\hline HAT-P-11 & 5.2 & 3.1 & 4785 & 12 & 0.262 & 0.004 & 0.8 & 0.01 & 4.59 & 0.02 & 0.75 & 0.01 & 0 & 1 \\
\hline HAT-P-13 & 5.6 & 0.9 & 5733 & 42 & 2.7 & 0.2 & 1.2 & 0.1 & 4.071 & 0.005 & 1.7 & 0.1 & 0 & 1 \\
\hline HAT-P-14 & 1.1 & 0.8 & 6694 & 73 & 4.3 & 0.3 & 1.42 & 0.04 & 4.21 & 0.03 & 1.54 & 0.08 & 0 & 1 \\
\hline HAT-P-17 & 8.9 & 2.9 & 5338 & 30 & 0.57 & 0.01 & 0.87 & 0.02 & 4.48 & 0.03 & 0.88 & 0.02 & 0 & 1 \\
\hline HAT-P-22 & 12.3 & 0.6 & 5338 & 14 & 0.75 & 0.01 & 0.9 & 0.01 & 4.37 & 0.01 & 1.02 & 0.01 & 0 & 1 \\
\hline HAT-P-24 & 2.2 & 1.2 & 6448 & 55 & 2.4 & 0.2 & 1.17 & 0.03 & 4.30 & 0.02 & 1.26 & 0.06 & 0 & 1 \\
\hline HAT-P-26 & 3.6 & 0.3 & 5387 & 6 & 0.53 & 0.01 & 0.9 & 0.002 & 4.541 & 0.004 & 0.84 & 0.01 & 0 & 1 \\
\hline HAT-P-27 & 7.9 & 2.1 & 5204 & 28 & 0.52 & 0.01 & 0.9 & 0.02 & 4.49 & 0.02 & 0.89 & 0.01 & 0 & 1 \\
\hline HAT-P-36 & 10.0 & 0.7 & 5422 & 23 & 0.83 & 0.01 & 0.94 & 0.01 & 4.37 & 0.01 & 1.04 & 0.02 & 0 & 1 \\
\hline HAT-P-4 & 5.4 & 0.9 & 5809 & 45 & 2.2 & 0.1 & 1.19 & 0.05 & 4.169 & 0.003 & 1.5 & 0.1 & 0 & 1 \\
\hline HAT-P-7 & 0.8 & 0.7 & 6707 & 53 & 5.9 & 0.7 & 1.57 & 0.04 & 4.12 & 0.06 & 1.8 & 0.1 & 0 & 1 \\
\hline HAT-P-9 & 1.8 & 0.4 & 6386 & 38 & 2.60 & 0.04 & 1.27 & 0.01 & 4.29 & 0.01 & 1.32 & 0.03 & 0 & 1 \\
\hline HATS-1 & 7.5 & 1.1 & 5705 & 32 & 0.95 & 0.01 & 0.94 & 0.01 & 4.4 & 0.01 & 1.00 & 0.02 & 0 & 1 \\
\hline HD 100655 & 9.4 & 2.1 & 4675 & 4 & 47 & 2 & 1.0 & 0.1 & 2.39 & 0.02 & 10.4 & 0.2 & 0 & 0 \\
\hline HD 100777 & 5.9 & 1.8 & 5579 & 37 & 0.92 & 0.02 & 1.00 & 0.02 & 4.41 & 0.03 & 1.03 & 0.03 & 0 & 0 \\
\hline HD 10180 & 4.5 & 1.1 & 5939 & 43 & 1.49 & 0.03 & 1.08 & 0.02 & 4.34 & 0.03 & 1.16 & 0.03 & 0 & 0 \\
\hline HD 101930 & 5.7 & 4.3 & 5144 & 27 & 0.42 & 0.02 & 0.87 & 0.03 & 4.54 & 0.04 & 0.82 & 0.02 & 1 & 0 \\
\hline HD 102117 & 6.1 & 1.2 & 5727 & 47 & 1.4 & 0.04 & 1.07 & 0.02 & 4.3 & 0.03 & 1.21 & 0.04 & 0 & 0 \\
\hline HD 102195 & 5.9 & 3.5 & 5283 & 29 & 0.49 & 0.01 & 0.88 & 0.03 & 4.53 & 0.03 & 0.84 & 0.02 & 0 & 0 \\
\hline HD 102272 & 11.6 & 1.2 & 4794 & 18 & 21.5 & 0.2 & 0.92 & 0.03 & 2.74 & 0.02 & 6.7 & 0.1 & 0 & 0 \\
\hline HD 102329 & 4.1 & 0.8 & 4786 & 39 & 19.0 & 0.3 & 1.4 & 0.1 & 2.96 & 0.04 & 6.4 & 0.2 & 0 & 0 \\
\hline HD 102365 & 10.8 & 2.0 & 5687 & 39 & 0.81 & 0.01 & 0.84 & 0.02 & 4.42 & 0.03 & 0.93 & 0.02 & 0 & 0 \\
\hline HD 102956 & 2.9 & 0.2 & 4892 & 14 & 11.9 & 0.1 & 1.54 & 0.02 & 3.25 & 0.01 & 4.8 & 0.1 & 0 & 0 \\
\hline HD 103197 & 3.4 & 2.0 & 5237 & 18 & 0.48 & 0.01 & 0.91 & 0.02 & 4.54 & 0.02 & 0.84 & 0.01 & 0 & 0 \\
\hline HD 103774 & 2.0 & 0.1 & 6391 & 27 & 3.7 & 0.1 & 1.38 & 0.01 & 4.183 & 0.002 & 1.56 & 0.03 & 0 & 0 \\
\hline HD 104067 & 9.0 & 3.4 & 4961 & 12 & 0.31 & 0.01 & 0.78 & 0.01 & 4.57 & 0.02 & 0.75 & 0.02 & 0 & 0 \\
\hline HD 104985 & 4.9 & 1.2 & 4730 & 41 & 51 & 1 & 1.2 & 0.1 & 2.5 & 0.1 & 10.6 & 0.3 & 0 & 0 \\
\hline HD 106252 & 6.4 & 1.4 & 5881 & 47 & 1.37 & 0.04 & 1.02 & 0.02 & 4.33 & 0.03 & 1.13 & 0.03 & 0 & 0 \\
\hline HD 106270 & 3.8 & 0.2 & 5570 & 123 & 5.9 & 0.3 & 1.37 & 0.03 & 3.74 & 0.05 & 2.6 & 0.2 & 0 & 0 \\
\hline HD 10647 & 1.8 & 0.9 & 6159 & 39 & 1.56 & 0.02 & 1.12 & 0.02 & 4.4 & 0.02 & 1.1 & 0.02 & 0 & 0 \\
\hline HD 10697 & 7.5 & 0.4 & 5674 & 93 & 2.8 & 0.04 & 1.12 & 0.01 & 4.00 & 0.03 & 1.7 & 0.1 & 0 & 0 \\
\hline HD 107148 & 4.0 & 1.0 & 5789 & 36 & 1.34 & 0.05 & 1.1 & 0.01 & 4.35 & 0.03 & 1.15 & 0.03 & 0 & 0 \\
\hline HD 108147 & 1.3 & 0.5 & 6211 & 21 & 1.88 & 0.02 & 1.21 & 0.01 & 4.37 & 0.01 & 1.19 & 0.01 & 0 & 0 \\
\hline HD 108863 & 3.3 & 0.5 & 4876 & 29 & 16.5 & 0.3 & 1.4 & 0.1 & 3.08 & 0.03 & 5.7 & 0.1 & 0 & 0 \\
\hline HD 108874 & 6.3 & 0.7 & 5647 & 19 & 1.06 & 0.01 & 1.02 & 0.01 & 4.37 & 0.01 & 1.08 & 0.01 & 0 & 0 \\
\hline HD 109246 & 2.5 & 0.8 & 5887 & 19 & 1.15 & 0.01 & 1.07 & 0.01 & 4.43 & 0.01 & 1.03 & 0.01 & 0 & 0 \\
\hline HD 109749 & 4.1 & 0.7 & 5860 & 39 & 1.55 & 0.02 & 1.14 & 0.01 & 4.32 & 0.02 & 1.21 & 0.02 & 1 & 0 \\
\hline HD 111232 & 11.6 & 1.5 & 5650 & 22 & 0.67 & 0.01 & 0.79 & 0.01 & 4.47 & 0.02 & 0.85 & 0.01 & 0 & 0 \\
\hline HD 113337 & 1.5 & 0.9 & 6622 & 71 & 4.18 & 0.08 & 1.38 & 0.03 & 4.19 & 0.03 & 1.56 & 0.05 & 0 & 0 \\
\hline HD 114386 & 8.8 & 2.8 & 4926 & 13 & 0.28 & 0.01 & 0.76 & 0.01 & 4.58 & 0.02 & 0.73 & 0.01 & 0 & 0 \\
\hline
\end{tabular}


Table A.1. continued.

\begin{tabular}{|c|c|c|c|c|c|c|c|c|c|c|c|c|c|c|}
\hline Star & $\begin{array}{r}{ }^{t} \\
\text { (Gyr) }\end{array}$ & $\begin{array}{r}\Delta t \\
(\mathrm{Gyr}) \\
\end{array}$ & $\begin{array}{l}T_{\text {eff }} \\
(\mathrm{K})\end{array}$ & $\begin{array}{r}\Delta T_{\text {eff }} \\
(\mathrm{K})\end{array}$ & $\begin{array}{r}L \\
\left(L_{\odot}\right)\end{array}$ & $\begin{array}{r}\Delta L \\
\left(L_{\odot}\right)\end{array}$ & $\begin{array}{r}M \\
\left(M_{\odot}\right) \\
\end{array}$ & $\begin{array}{r}\Delta M \\
\left(M_{\odot}\right)\end{array}$ & $\begin{array}{r}\log g \\
\left(\mathrm{~cm} / \mathrm{s}^{2}\right)\end{array}$ & $\begin{array}{r}\Delta \log g \\
\left(\mathrm{~cm} / \mathrm{s}^{2}\right)\end{array}$ & $\begin{array}{r}R \\
\left(R_{\odot}\right) \\
\end{array}$ & $\begin{array}{r}\Delta R \\
\left(R_{\odot}\right)\end{array}$ & Bin & $\operatorname{Tr}$ \\
\hline HD 114613 & 5.0 & 0.1 & 5756 & 13 & 4.09 & 0.03 & 1.25 & 0.01 & 3.91 & 0.01 & 2.04 & 0.02 & 0 & 0 \\
\hline HD 114729 & 9.3 & 0.6 & 5939 & 58 & 2.33 & 0.02 & 0.97 & 0.01 & 4.1 & 0.02 & 1.44 & 0.03 & 1 & 0 \\
\hline HD 114762 & 12.4 & 0.6 & 6043 & 22 & 1.52 & 0.01 & 0.82 & 0.01 & 4.24 & 0.01 & 1.13 & 0.01 & 1 & 0 \\
\hline HD 114783 & 10.4 & 2.4 & 5089 & 17 & 0.4 & 0.01 & 0.81 & 0.01 & 4.53 & 0.02 & 0.81 & 0.01 & 0 & 0 \\
\hline HD 11506 & 2.0 & 0.6 & 6119 & 45 & 2.1 & 0.1 & 1.25 & 0.01 & 4.31 & 0.02 & 1.29 & 0.04 & 0 & 0 \\
\hline HD 116029 & 4.9 & 1.1 & 4849 & 39 & 11.4 & 0.1 & 1.3 & 0.1 & 3.19 & 0.04 & 4.8 & 0.1 & 0 & 0 \\
\hline HD 117207 & 6.6 & 1.0 & 5681 & 33 & 1.19 & 0.02 & 1.03 & 0.01 & 4.34 & 0.02 & 1.13 & 0.02 & 0 & 0 \\
\hline HD 117618 & 4.0 & 1.3 & 6019 & 50 & 1.6 & 0.1 & 1.1 & 0.02 & 4.34 & 0.03 & 1.17 & 0.04 & 0 & 0 \\
\hline HD 118203 & 5.4 & 0.5 & 5741 & 35 & 3.8 & 0.3 & 1.23 & 0.03 & 3.93 & 0.02 & 2.0 & 0.1 & 0 & 0 \\
\hline HD 11964 & 8.5 & 0.5 & 5371 & 43 & 2.6 & 0.1 & 1.09 & 0.02 & 3.92 & 0.02 & 1.9 & 0.1 & 1 & 0 \\
\hline HD 11977 & 2.9 & 0.2 & 4851 & 9 & 62.1 & 0.2 & 1.46 & 0.03 & 2.5 & 0.01 & 11.2 & 0.1 & 0 & 0 \\
\hline HD 120084 & 4.4 & 1.2 & 4675 & 11 & 52 & 1 & 1.3 & 0.1 & 2.48 & 0.03 & 11.0 & 0.2 & 0 & 0 \\
\hline HD 121504 & 1.9 & 1.0 & 6089 & 47 & 1.62 & 0.04 & 1.16 & 0.02 & 4.38 & 0.03 & 1.15 & 0.03 & 0 & 0 \\
\hline HD 125595 & 8.0 & 3.7 & 4654 & 22 & 0.21 & 0.01 & 0.74 & 0.01 & 4.61 & 0.02 & 0.71 & 0.02 & 0 & 0 \\
\hline HD 125612 & 3.1 & 0.3 & 5818 & 13 & 1.205 & 0.003 & 1.09 & 0.01 & 4.4 & 0.01 & 1.08 & 0.01 & 1 & 0 \\
\hline HD 12661 & 3.3 & 0.6 & 5714 & 22 & 1.13 & 0.01 & 1.09 & 0.01 & 4.4 & 0.01 & 1.08 & 0.01 & 0 & 0 \\
\hline HD 128311 & 8.5 & 3.6 & 4922 & 26 & 0.29 & 0.01 & 0.77 & 0.02 & 4.58 & 0.02 & 0.74 & 0.02 & 0 & 0 \\
\hline HD 130322 & 6.1 & 2.9 & 5410 & 30 & 0.56 & 0.01 & 0.88 & 0.02 & 4.52 & 0.03 & 0.85 & 0.02 & 0 & 0 \\
\hline HD 131496 & 4.0 & 0.7 & 4838 & 43 & 9.9 & 0.2 & 1.4 & 0.1 & 3.27 & 0.04 & 4.5 & 0.1 & 0 & 0 \\
\hline HD 134987 & 5.4 & 0.5 & 5797 & 23 & 1.51 & 0.01 & 1.09 & 0.01 & 4.3 & 0.01 & 1.22 & 0.01 & 0 & 0 \\
\hline HD 136418 & 5.0 & 1.0 & 4997 & 40 & 6.9 & 0.1 & 1.2 & 0.1 & 3.43 & 0.04 & 3.5 & 0.1 & 0 & 0 \\
\hline HD 137388 & 7.3 & 3.8 & 5183 & 25 & 0.46 & 0.02 & 0.87 & 0.03 & 4.52 & 0.03 & 0.85 & 0.02 & 1 & 0 \\
\hline HD 13908 & 3.9 & 0.5 & 6212 & 38 & 4.0 & 0.1 & 1.28 & 0.04 & 4.06 & 0.02 & 1.74 & 0.04 & 0 & 0 \\
\hline HD 13931 & 5.3 & 1.3 & 5902 & 52 & 1.48 & 0.03 & 1.07 & 0.02 & 4.33 & 0.03 & 1.17 & 0.03 & 0 & 0 \\
\hline HD 139357 & 7.2 & 1.8 & 4454 & 39 & 73.5 & 1.3 & 1.1 & 0.1 & 2.2 & 0.1 & 14.4 & 0.4 & 0 & 0 \\
\hline HD 141937 & 3.2 & 0.5 & 5837 & 14 & 1.13 & 0.01 & 1.06 & 0.01 & 4.42 & 0.01 & 1.04 & 0.01 & 0 & 0 \\
\hline HD 142 & 2.8 & 0.5 & 6321 & 67 & 2.81 & 0.05 & 1.25 & 0.01 & 4.24 & 0.03 & 1.4 & 0.04 & 1 & 0 \\
\hline HD 142245 & 3.1 & 0.3 & 4831 & 28 & 13.1 & 0.2 & 1.52 & 0.05 & 3.19 & 0.03 & 5.2 & 0.1 & 0 & 0 \\
\hline HD 142415 & 1.6 & 0.6 & 5869 & 12 & 1.16 & 0.02 & 1.1 & 0.01 & 4.44 & 0.01 & 1.04 & 0.01 & 0 & 0 \\
\hline HD 145377 & 2.9 & 1.2 & 5979 & 46 & 1.43 & 0.04 & 1.11 & 0.02 & 4.38 & 0.03 & 1.12 & 0.03 & 0 & 0 \\
\hline HD 145457 & 2.8 & 0.6 & 4772 & 45 & 41 & 1 & 1.5 & 0.1 & 2.66 & 0.05 & 9.4 & 0.2 & 0 & 0 \\
\hline HD 1461 & 4.0 & 0.7 & 5807 & 20 & 1.20 & 0.01 & 1.07 & 0.01 & 4.39 & 0.01 & 1.08 & 0.01 & 0 & 0 \\
\hline HD 147018 & 7.3 & 2.0 & 5526 & 29 & 0.78 & 0.02 & 0.94 & 0.02 & 4.44 & 0.03 & 0.97 & 0.02 & 0 & 0 \\
\hline HD 147513 & 3.4 & 0.7 & 5827 & 21 & 1.01 & 0.01 & 1.02 & 0.01 & 4.45 & 0.01 & 0.99 & 0.01 & 1 & 0 \\
\hline HD 148156 & 1.2 & 0.5 & 6156 & 23 & 1.84 & 0.03 & 1.22 & 0.01 & 4.36 & 0.01 & 1.19 & 0.02 & 0 & 0 \\
\hline HD 148427 & 4.3 & 0.6 & 4993 & 44 & 6.2 & 0.1 & 1.32 & 0.05 & 3.51 & 0.03 & 3.3 & 0.1 & 0 & 0 \\
\hline HD 149026 & 2.9 & 0.3 & 6116 & 44 & 2.8 & 0.1 & 1.302 & 0.005 & 4.2 & 0.02 & 1.49 & 0.04 & 0 & 1 \\
\hline HD 149143 & 4.8 & 0.8 & 5792 & 58 & 2.2 & 0.1 & 1.21 & 0.03 & 4.17 & 0.03 & 1.5 & 0.1 & 0 & 0 \\
\hline HD 1502 & 3.0 & 0.3 & 5006 & 25 & 11.5 & 0.2 & 1.46 & 0.04 & 3.29 & 0.02 & 4.5 & 0.1 & 0 & 0 \\
\hline HD 152581 & 7.2 & 2.0 & 4991 & 45 & 16.1 & 0.2 & 1.0 & 0.1 & 3.0 & 0.1 & 5.4 & 0.1 & 0 & 0 \\
\hline HD 153950 & 4.3 & 0.8 & 6136 & 64 & 2.24 & 0.03 & 1.15 & 0.02 & 4.25 & 0.03 & 1.33 & 0.04 & 0 & 0 \\
\hline HD 154345 & 4.1 & 1.2 & 5557 & 15 & 0.62 & 0.002 & 0.9 & 0.01 & 4.53 & 0.01 & 0.85 & 0.01 & 0 & 0 \\
\hline HD 154672 & 7.1 & 0.8 & 5754 & 51 & 1.81 & 0.02 & 1.09 & 0.02 & 4.2 & 0.03 & 1.36 & 0.03 & 0 & 0 \\
\hline HD 154857 & 5.8 & 0.5 & 5740 & 46 & 4.4 & 0.3 & 1.13 & 0.03 & 3.83 & 0.03 & 2.1 & 0.1 & 0 & 0 \\
\hline HD 155358 & 1.9 & 4.5 & 5966 & 53 & 2.11 & 0.02 & 1.1 & 0.1 & 4.2 & 0.04 & 1.36 & 0.03 & 0 & 0 \\
\hline HD 156279 & 7.4 & 2.2 & 5449 & 31 & 0.7 & 0.01 & 0.93 & 0.02 & 4.45 & 0.03 & 0.94 & 0.02 & 0 & 0 \\
\hline HD 156411 & 4.5 & 0.3 & 5886 & 29 & 5.1 & 0.3 & 1.23 & 0.02 & 3.85 & 0.02 & 2.2 & 0.1 & 0 & 0 \\
\hline HD 156668 & 10.2 & 2.8 & 4857 & 18 & 0.27 & 0.01 & 0.75 & 0.01 & 4.58 & 0.01 & 0.73 & 0.01 & 0 & 0 \\
\hline HD 158038 & 3.2 & 0.4 & 4839 & 29 & 11.9 & 0.1 & 1.5 & 0.1 & 3.23 & 0.03 & 4.9 & 0.1 & 0 & 0 \\
\hline HD 159243 & 2.0 & 0.3 & 6071 & 19 & 1.45 & 0.05 & 1.12 & 0.01 & 4.4 & 0.01 & 1.09 & 0.03 & 0 & 0 \\
\hline HD 159868 & 6.3 & 0.5 & 5583 & 54 & 3.8 & 0.2 & 1.13 & 0.03 & 3.85 & 0.03 & 2.1 & 0.1 & 0 & 0 \\
\hline HD 16141 & 6.5 & 0.6 & 5856 & 60 & 2.46 & 0.03 & 1.13 & 0.02 & 4.12 & 0.03 & 1.53 & 0.04 & 1 & 0 \\
\hline HD 16175 & 4.1 & 0.8 & 6009 & 44 & 3.35 & 0.02 & 1.3 & 0.05 & 4.09 & 0.02 & 1.69 & 0.03 & 0 & 0 \\
\hline HD 162020 & 3.1 & 2.7 & 4807 & 17 & 0.22 & 0.01 & 0.75 & 0.01 & 4.63 & 0.01 & 0.68 & 0.01 & 0 & 0 \\
\hline HD 163607 & 8.3 & 0.5 & 5508 & 15 & 2.6 & 0.1 & 1.1 & 0.02 & 3.98 & 0.01 & 1.8 & 0.1 & 0 & 0 \\
\hline HD 16417 & 6.9 & 0.4 & 5818 & 51 & 2.74 & 0.01 & 1.12 & 0.01 & 4.06 & 0.02 & 1.63 & 0.03 & 0 & 0 \\
\hline HD 164509 & 3.2 & 0.8 & 5860 & 31 & 1.31 & 0.02 & 1.1 & 0.01 & 4.38 & 0.02 & 1.11 & 0.02 & 0 & 0 \\
\hline HD 164922 & 7.9 & 2.7 & 5439 & 38 & 0.7 & 0.01 & 0.93 & 0.02 & 4.45 & 0.03 & 0.95 & 0.02 & 0 & 0 \\
\hline HD 167042 & 3.1 & 0.3 & 4989 & 32 & 10.7 & 0.1 & 1.46 & 0.05 & 3.31 & 0.03 & 4.4 & 0.1 & 0 & 0 \\
\hline HD 168443 & 10.0 & 0.3 & 5646 & 36 & 2.08 & 0.01 & 1.02 & 0.01 & 4.08 & 0.01 & 1.51 & 0.02 & 0 & 0 \\
\hline HD 168746 & 12.0 & 0.9 & 5637 & 26 & 1.04 & 0.01 & 0.9 & 0.01 & 4.32 & 0.01 & 1.07 & 0.01 & 0 & 0 \\
\hline HD 169830 & 2.82 & 0.03 & 6276 & 12 & 4.656 & 0.003 & 1.3975 & $4.0 \mathrm{E}-4$ & 4.052 & 0.004 & 1.83 & 0.01 & 0 & 0 \\
\hline HD 170469 & 4.7 & 0.9 & 5866 & 54 & 1.7 & 0.1 & 1.14 & 0.01 & 4.28 & 0.03 & 1.27 & 0.05 & 0 & 0 \\
\hline HD 171028 & 8.2 & 1.1 & 5771 & 46 & 3.9 & 0.5 & 0.98 & 0.04 & 3.84 & 0.03 & 2.0 & 0.2 & 0 & 0 \\
\hline HD 171238 & 4.0 & 1.2 & 5570 & 21 & 0.774 & 0.003 & 0.99 & 0.01 & 4.47 & 0.01 & 0.95 & 0.01 & 0 & 0 \\
\hline
\end{tabular}


Table A.1. continued.

\begin{tabular}{|c|c|c|c|c|c|c|c|c|c|c|c|c|c|c|}
\hline Star & $\begin{array}{r}t \\
(\mathrm{Gyr})\end{array}$ & $\begin{array}{r}\Delta t \\
(\mathrm{Gyr}) \\
\end{array}$ & $\begin{array}{l}T_{\text {eff }} \\
(\mathrm{K})\end{array}$ & $\begin{array}{r}\Delta T_{\text {eff }} \\
(\mathrm{K})\end{array}$ & $\begin{array}{r}L \\
\left(L_{\odot}\right)\end{array}$ & $\begin{array}{r}\Delta L \\
\left(L_{\odot}\right)\end{array}$ & $\begin{array}{r}M \\
\left(M_{\odot}\right)\end{array}$ & $\begin{array}{r}\Delta M \\
\left(M_{\odot}\right)\end{array}$ & $\begin{array}{r}\log g \\
\left(\mathrm{~cm} / \mathrm{s}^{2}\right)\end{array}$ & $\begin{array}{r}\Delta \log g \\
\left(\mathrm{~cm} / \mathrm{s}^{2}\right)\end{array}$ & $\begin{array}{r}R \\
\left(R_{\odot}\right) \\
\end{array}$ & $\begin{array}{r}\Delta R \\
\left(R_{\odot}\right)\end{array}$ & Bin & $\operatorname{Tr}$ \\
\hline HD 17156 & 4.9 & 0.6 & 5943 & 38 & 2.46 & 0.05 & 1.2 & 0.03 & 4.17 & 0.02 & 1.48 & 0.03 & 0 & 1 \\
\hline HD 173416 & 1.8 & 0.7 & 4790 & 37 & 80 & 2 & 1.8 & 0.2 & 2.5 & 0.1 & 13.0 & 0.3 & 0 & 0 \\
\hline HD 175541 & 2.9 & 0.2 & 5093 & 23 & 10.0 & 0.1 & 1.45 & 0.03 & 3.37 & 0.02 & 4.07 & 0.05 & 0 & 0 \\
\hline HD 177830 & 10.2 & 1.7 & 4735 & 31 & 5.3 & 0.1 & 1.1 & 0.1 & 3.39 & 0.04 & 3.4 & 0.1 & 1 & 0 \\
\hline HD $178911 \mathrm{~B}$ & 4.8 & 1.3 & 5642 & 29 & 1.00 & 0.02 & 1.03 & 0.02 & 4.4 & 0.02 & 1.05 & 0.02 & 1 & 0 \\
\hline HD 179079 & 7.8 & 0.4 & 5649 & 47 & 2.392 & 0.004 & 1.11 & 0.01 & 4.06 & 0.02 & 1.62 & 0.03 & 0 & 0 \\
\hline HD 179949 & 1.2 & 0.6 & 6220 & 28 & 1.95 & 0.01 & 1.23 & 0.01 & 4.36 & 0.01 & 1.2 & 0.01 & 0 & 0 \\
\hline HD 180314 & 0.9 & 0.2 & 4946 & 55 & 40 & 1 & 2.3 & 0.1 & 2.92 & 0.05 & 8.7 & 0.3 & 0 & 0 \\
\hline HD 180902 & 3.3 & 0.5 & 5001 & 44 & 9.4 & 0.1 & 1.4 & 0.1 & 3.36 & 0.04 & 4.1 & 0.1 & 0 & 0 \\
\hline HD 181342 & 3.6 & 0.6 & 4856 & 41 & 12.3 & 0.4 & 1.4 & 0.1 & 3.2 & 0.04 & 5.0 & 0.2 & 0 & 0 \\
\hline HD 181433 & 7.4 & 3.4 & 4909 & 20 & 0.34 & 0.01 & 0.84 & 0.02 & 4.55 & 0.02 & 0.8 & 0.02 & 0 & 0 \\
\hline HD 181720 & 12.4 & 0.5 & 5840 & 49 & 2.112 & 0.003 & 0.87 & 0.01 & 4.06 & 0.02 & 1.42 & 0.02 & 0 & 0 \\
\hline HD 183263 & 4.5 & 0.8 & 5870 & 56 & 1.8 & 0.1 & 1.16 & 0.02 & 4.28 & 0.03 & 1.29 & 0.05 & 0 & 0 \\
\hline HD 185269 & 4.1 & 0.5 & 6023 & 43 & 4.5 & 0.1 & 1.3 & 0.04 & 3.97 & 0.03 & 2.0 & 0.1 & 1 & 0 \\
\hline HD 187085 & 2.7 & 0.8 & 6163 & 53 & 2.0 & 0.1 & 1.19 & 0.02 & 4.31 & 0.03 & 1.26 & 0.04 & 0 & 0 \\
\hline HD 187123 & 5.6 & 1.3 & 5853 & 53 & 1.44 & 0.02 & 1.06 & 0.02 & 4.32 & 0.03 & 1.17 & 0.03 & 0 & 0 \\
\hline HD 18742 & 3.9 & 0.8 & 4956 & 40 & 14.0 & 0.2 & 1.3 & 0.1 & 3.14 & 0.04 & 5.1 & 0.1 & 0 & 0 \\
\hline HD 188015 & 5.9 & 1.3 & 5722 & 52 & 1.41 & 0.03 & 1.08 & 0.02 & 4.3 & 0.03 & 1.21 & 0.03 & 1 & 0 \\
\hline HD 189733 & 5.3 & 3.8 & 5019 & 23 & 0.327 & 0.003 & 0.81 & 0.02 & 4.58 & 0.02 & 0.76 & 0.01 & 1 & 1 \\
\hline HD 190360 & 7.3 & 1.6 & 5628 & 47 & 1.12 & 0.03 & 1.01 & 0.02 & 4.34 & 0.03 & 1.12 & 0.03 & 1 & 0 \\
\hline HD 190647 & 8.7 & 0.4 & 5630 & 48 & 2.19 & 0.01 & 1.07 & 0.01 & 4.07 & 0.02 & 1.56 & 0.03 & 0 & 0 \\
\hline HD 192263 & 5.9 & 3.9 & 4980 & 20 & 0.3 & 0.01 & 0.78 & 0.02 & 4.59 & 0.02 & 0.73 & 0.01 & 0 & 0 \\
\hline HD 192310 & 8.1 & 3.2 & 5153 & 21 & 0.4 & 0.01 & 0.82 & 0.02 & 4.54 & 0.02 & 0.79 & 0.01 & 0 & 0 \\
\hline HD 192699 & 3.1 & 0.4 & 5097 & 36 & 11.1 & 0.1 & 1.39 & 0.05 & 3.31 & 0.03 & 4.3 & 0.1 & 0 & 0 \\
\hline HD 195019 & 7.7 & 0.7 & 5825 & 56 & 2.23 & 0.02 & 1.08 & 0.01 & 4.13 & 0.02 & 1.47 & 0.04 & 1 & 0 \\
\hline HD 196050 & 5.4 & 0.7 & 5884 & 47 & 2.09 & 0.02 & 1.15 & 0.02 & 4.2 & 0.02 & 1.4 & 0.03 & 1 & 0 \\
\hline HD 19994 & 3.1 & 0.3 & 6164 & 62 & 3.78 & 0.04 & 1.35 & 0.01 & 4.1 & 0.02 & 1.71 & 0.04 & 1 & 0 \\
\hline HD 200964 & 3.1 & 0.4 & 5059 & 34 & 12.8 & 0.2 & 1.4 & 0.1 & 3.23 & 0.03 & 4.7 & 0.1 & 0 & 0 \\
\hline HD 202206 & 2.9 & 1.0 & 5719 & 26 & 1.04 & 0.01 & 1.07 & 0.02 & 4.43 & 0.02 & 1.04 & 0.01 & 0 & 0 \\
\hline HD 2039 & 4.4 & 0.9 & 5927 & 60 & 2.1 & 0.1 & 1.19 & 0.02 & 4.23 & 0.04 & 1.4 & 0.1 & 0 & 0 \\
\hline HD 204313 & 4.3 & 1.8 & 5783 & 48 & 1.18 & 0.03 & 1.06 & 0.03 & 4.39 & 0.04 & 1.08 & 0.03 & 0 & 0 \\
\hline HD 204941 & 3.9 & 3.3 & 5072 & 17 & 0.297 & 0.003 & 0.77 & 0.02 & 4.62 & 0.02 & 0.71 & 0.01 & 1 & 0 \\
\hline HD 205739 & 2.9 & 0.2 & 6247 & 40 & 3.581 & 0.003 & 1.335 & 0.003 & 4.14 & 0.01 & 1.62 & 0.02 & 0 & 0 \\
\hline HD 206610 & 3.0 & 0.3 & 4836 & 30 & 18 & 1 & 1.51 & 0.05 & 3.05 & 0.03 & 6.0 & 0.2 & 0 & 0 \\
\hline HD 20782 & 5.4 & 1.3 & 5876 & 31 & 1.20 & 0.03 & 1.01 & 0.02 & 4.39 & 0.03 & 1.06 & 0.03 & 1 & 0 \\
\hline HD 207832 & 1.4 & 0.8 & 5676 & 40 & 0.82 & 0.04 & 1.03 & 0.02 & 4.5 & 0.01 & 0.94 & 0.04 & 0 & 0 \\
\hline HD 20794 & 11.6 & 1.5 & 5602 & 20 & 0.642 & 0.003 & 0.8 & 0.01 & 4.47 & 0.02 & 0.85 & 0.01 & 0 & 0 \\
\hline HD 208487 & 2.3 & 0.9 & 6143 & 47 & 1.76 & 0.05 & 1.16 & 0.02 & 4.36 & 0.03 & 1.17 & 0.03 & 0 & 0 \\
\hline HD 20868 & 8.2 & 2.7 & 4769 & 24 & 0.25 & 0.01 & 0.77 & 0.01 & 4.59 & 0.01 & 0.73 & 0.02 & 0 & 0 \\
\hline HD 209458 & 4.4 & 1.2 & 6047 & 62 & 1.8 & 0.04 & 1.11 & 0.02 & 4.3 & 0.04 & 1.22 & 0.04 & 0 & 1 \\
\hline HD 210277 & 8.8 & 1.9 & 5530 & 40 & 0.92 & 0.03 & 0.96 & 0.02 & 4.37 & 0.03 & 1.05 & 0.03 & 0 & 0 \\
\hline HD 210702 & 3.1 & 0.3 & 4946 & 25 & 12.9 & 0.1 & 1.47 & 0.04 & 3.22 & 0.02 & 4.9 & 0.1 & 0 & 0 \\
\hline HD 212771 & 2.9 & 0.1 & 5008 & 14 & 15.1 & 0.2 & 1.45 & 0.02 & 3.16 & 0.02 & 5.2 & 0.1 & 0 & 0 \\
\hline HD 213240 & 4.6 & 0.6 & 6029 & 37 & 2.6 & 0.1 & 1.2 & 0.02 & 4.17 & 0.02 & 1.48 & 0.03 & 1 & 0 \\
\hline HD 215497 & 9.9 & 2.8 & 5128 & 12 & 0.47 & 0.02 & 0.86 & 0.02 & 4.49 & 0.03 & 0.87 & 0.02 & 0 & 0 \\
\hline HD 216437 & 5.2 & 0.7 & 5898 & 37 & 2.23 & 0.03 & 1.17 & 0.03 & 4.19 & 0.02 & 1.43 & 0.03 & 0 & 0 \\
\hline HD 216770 & 5.4 & 2.9 & 5406 & 39 & 0.66 & 0.01 & 0.95 & 0.03 & 4.48 & 0.03 & 0.93 & 0.02 & 0 & 0 \\
\hline HD 217107 & 4.2 & 1.0 & 5676 & 31 & 1.14 & 0.01 & 1.08 & 0.01 & 4.38 & 0.02 & 1.11 & 0.02 & 0 & 0 \\
\hline HD 217786 & 6.8 & 0.9 & 6031 & 55 & 1.93 & 0.04 & 1.03 & 0.02 & 4.23 & 0.03 & 1.27 & 0.04 & 0 & 0 \\
\hline HD 218566 & 8.0 & 3.1 & 4880 & 16 & 0.3 & 0.01 & 0.8 & 0.01 & 4.57 & 0.02 & 0.77 & 0.02 & 0 & 0 \\
\hline HD 219828 & 5.2 & 0.8 & 5921 & 53 & 2.74 & 0.03 & 1.2 & 0.04 & 4.11 & 0.03 & 1.58 & 0.04 & 0 & 0 \\
\hline HD 220773 & 6.3 & 0.1 & 5852 & 26 & 3.16 & 0.01 & 1.154 & 0.003 & 4.02 & 0.01 & 1.73 & 0.02 & 0 & 0 \\
\hline HD 221287 & 2.8 & 0.3 & 6193 & 20 & 2.0 & 0.1 & 1.17 & 0.01 & 4.33 & 0.01 & 1.22 & 0.03 & 0 & 0 \\
\hline HD 222155 & 8.1 & 0.4 & 5814 & 43 & 2.9 & 0.1 & 1.05 & 0.01 & 4.00 & 0.01 & 1.7 & 0.1 & 0 & 0 \\
\hline HD 222582 & 6.2 & 1.1 & 5851 & 32 & 1.24 & 0.01 & 1.01 & 0.02 & 4.36 & 0.02 & 1.09 & 0.02 & 1 & 0 \\
\hline HD 224693 & 3.9 & 0.5 & 5972 & 49 & 4.1 & 0.1 & 1.35 & 0.04 & 4.01 & 0.01 & 1.89 & 0.05 & 0 & 0 \\
\hline HD 22781 & 7.5 & 2.9 & 5152 & 27 & 0.32 & 0.01 & 0.74 & 0.02 & 4.6 & 0.02 & 0.71 & 0.02 & 0 & 0 \\
\hline HD 23079 & 4.1 & 1.4 & 6039 & 44 & 1.37 & 0.03 & 1.03 & 0.02 & 4.39 & 0.03 & 1.07 & 0.03 & 0 & 0 \\
\hline HD 23127 & 4.4 & 0.6 & 5841 & 45 & 3.08 & 0.02 & 1.29 & 0.03 & 4.07 & 0.02 & 1.72 & 0.03 & 0 & 0 \\
\hline HD 231701 & 3.7 & 0.5 & 6211 & 71 & 2.94 & 0.05 & 1.23 & 0.01 & 4.18 & 0.03 & 1.48 & 0.05 & 0 & 0 \\
\hline HD 23596 & 4.0 & 0.7 & 5979 & 68 & 2.65 & 0.03 & 1.25 & 0.03 & 4.17 & 0.03 & 1.52 & 0.04 & 0 & 0 \\
\hline HD 24040 & 4.9 & 0.9 & 5910 & 53 & 1.78 & 0.05 & 1.13 & 0.02 & 4.27 & 0.03 & 1.27 & 0.04 & 0 & 0 \\
\hline HD 25171 & 4.8 & 0.9 & 6131 & 57 & 1.94 & 0.02 & 1.08 & 0.02 & 4.28 & 0.03 & 1.24 & 0.03 & 0 & 0 \\
\hline HD 2638 & 5.1 & 4.1 & 5173 & 26 & 0.42 & 0.01 & 0.87 & 0.03 & 4.55 & 0.03 & 0.81 & 0.02 & 0 & 0 \\
\hline HD 27894 & 6.9 & 4.3 & 4923 & 32 & 0.33 & 0.01 & 0.83 & 0.03 & 4.56 & 0.03 & 0.79 & 0.02 & 0 & 0 \\
\hline
\end{tabular}


Table A.1. continued.

\begin{tabular}{|c|c|c|c|c|c|c|c|c|c|c|c|c|c|c|}
\hline Star & $\begin{array}{r}t \\
(\mathrm{Gyr})\end{array}$ & $\begin{array}{r}\Delta t \\
(\mathrm{Gyr})\end{array}$ & $\begin{array}{l}T_{\text {eff }} \\
(\mathrm{K})\end{array}$ & $\begin{array}{r}\Delta T_{\text {eff }} \\
(\mathrm{K})\end{array}$ & $\begin{array}{r}L \\
\left(L_{\odot}\right)\end{array}$ & $\begin{array}{r}\Delta L \\
\left(L_{\odot}\right)\end{array}$ & $\begin{array}{r}M \\
\left(M_{\odot}\right)\end{array}$ & $\begin{array}{r}\Delta M \\
\left(M_{\odot}\right)\end{array}$ & $\begin{array}{r}\log g \\
\left(\mathrm{~cm} / \mathrm{s}^{2}\right)\end{array}$ & $\begin{array}{r}\Delta \log g \\
\left(\mathrm{~cm} / \mathrm{s}^{2}\right)\end{array}$ & $\begin{array}{r}R \\
\left(R_{\odot}\right) \\
\end{array}$ & $\begin{array}{r}\Delta R \\
\left(R_{\odot}\right)\end{array}$ & Bin & $\operatorname{Tr}$ \\
\hline HD 28185 & 5.5 & 4.2 & 5615 & 56 & 1.17 & 0.02 & 1.0 & 0.1 & 4.33 & 0.03 & 1.15 & 0.03 & 0 & 0 \\
\hline HD 28254 & 7.8 & 0.4 & 5607 & 37 & 2.19 & 0.01 & 1.11 & 0.01 & 4.08 & 0.02 & 1.57 & 0.02 & 1 & 0 \\
\hline HD 28678 & 6.1 & 1.7 & 4798 & 43 & 24.0 & 0.4 & 1.1 & 0.1 & 2.8 & 0.1 & 7.1 & 0.2 & 0 & 0 \\
\hline HD 290327 & 11.5 & 1.3 & 5543 & 13 & 0.74 & 0.02 & 0.85 & 0.01 & 4.42 & 0.02 & 0.93 & 0.02 & 0 & 0 \\
\hline HD 2952 & 3.1 & 0.3 & 4755 & 18 & 61.5 & 0.4 & 1.5 & 0.1 & 2.47 & 0.02 & 11.6 & 0.1 & 0 & 0 \\
\hline HD 30177 & 5.9 & 1.1 & 5596 & 32 & 1.09 & 0.01 & 1.05 & 0.01 & 4.36 & 0.02 & 1.11 & 0.02 & 0 & 0 \\
\hline HD 30562 & 3.7 & 0.5 & 6000 & 55 & 2.8 & 0.02 & 1.28 & 0.02 & 4.16 & 0.02 & 1.55 & 0.03 & 0 & 0 \\
\hline HD 30856 & 7.3 & 1.8 & 4911 & 41 & 10.0 & 0.2 & 1.1 & 0.1 & 3.19 & 0.05 & 4.4 & 0.1 & 0 & 0 \\
\hline HD 31253 & 4.0 & 0.7 & 6105 & 63 & 2.9 & 0.1 & 1.25 & 0.04 & 4.16 & 0.03 & 1.5 & 0.1 & 0 & 0 \\
\hline HD 32518 & 5.8 & 1.5 & 4610 & 40 & 47 & 1 & 1.2 & 0.1 & 2.4 & 0.1 & 10.8 & 0.3 & 0 & 0 \\
\hline HD 330075 & 6.1 & 4.0 & 5127 & 26 & 0.4 & 0.03 & 0.84 & 0.02 & 4.55 & 0.03 & 0.8 & 0.04 & 0 & 0 \\
\hline HD 33142 & 3.3 & 0.4 & 4980 & 37 & 10.5 & 0.2 & 1.4 & 0.1 & 3.31 & 0.03 & 4.4 & 0.1 & 0 & 0 \\
\hline HD 33283 & 3.9 & 0.6 & 5980 & 54 & 4.43 & 0.02 & 1.37 & 0.04 & 3.98 & 0.03 & 1.97 & 0.04 & 0 & 0 \\
\hline HD 34445 & 3.7 & 0.6 & 6038 & 53 & 2.1 & 0.1 & 1.19 & 0.01 & 4.27 & 0.03 & 1.32 & 0.04 & 1 & 0 \\
\hline HD 3651 & 6.9 & 2.8 & 5271 & 26 & 0.51 & 0.01 & 0.88 & 0.02 & 4.51 & 0.02 & 0.86 & 0.01 & 1 & 0 \\
\hline HD 37124 & 11.1 & 1.7 & 5733 & 37 & 0.81 & 0.01 & 0.82 & 0.02 & 4.42 & 0.02 & 0.92 & 0.02 & 0 & 0 \\
\hline HD 37605 & 4.2 & 1.4 & 5364 & 25 & 0.62 & 0.01 & 0.96 & 0.01 & 4.49 & 0.01 & 0.91 & 0.02 & 0 & 0 \\
\hline HD 38283 & 6.5 & 0.6 & 6080 & 59 & 2.56 & 0.01 & 1.07 & 0.02 & 4.14 & 0.03 & 1.45 & 0.03 & 0 & 0 \\
\hline HD 38529 & 3.98 & 0.03 & 5526 & 17 & 5.81 & 0.03 & 1.412 & 0.003 & 3.74 & 0.01 & 2.64 & 0.02 & 1 & 0 \\
\hline HD 38801 & 4.8 & 0.3 & 5323 & 52 & 3.7 & 0.1 & 1.28 & 0.02 & 3.82 & 0.02 & 2.3 & 0.1 & 0 & 0 \\
\hline HD 39091 & 2.8 & 0.8 & 6018 & 31 & 1.5 & 0.02 & 1.12 & 0.01 & 4.37 & 0.02 & 1.13 & 0.02 & 0 & 0 \\
\hline HD 40307 & 6.9 & 4.0 & 4956 & 18 & 0.243 & 0.003 & 0.71 & 0.02 & 4.63 & 0.02 & 0.67 & 0.01 & 0 & 0 \\
\hline HD 40979 & 1.5 & 0.6 & 6163 & 25 & 1.82 & 0.03 & 1.21 & 0.01 & 4.36 & 0.02 & 1.19 & 0.02 & 1 & 0 \\
\hline HD 4113 & 5.8 & 1.6 & 5717 & 46 & 1.16 & 0.04 & 1.03 & 0.02 & 4.36 & 0.03 & 1.1 & 0.03 & 1 & 0 \\
\hline HD 4203 & 7.3 & 0.9 & 5640 & 57 & 1.71 & 0.02 & 1.09 & 0.02 & 4.2 & 0.03 & 1.37 & 0.04 & 0 & 0 \\
\hline HD 4208 & 7.4 & 2.4 & 5678 & 33 & 0.71 & 0.01 & 0.86 & 0.02 & 4.48 & 0.03 & 0.88 & 0.02 & 0 & 0 \\
\hline HD 4308 & 0.4 & 2.2 & 5674 & 51 & 1.02 & 0.03 & 0.96 & 0.03 & 4.37 & 0.02 & 1.05 & 0.03 & 0 & 0 \\
\hline HD 4313 & 3.0 & 0.3 & 4920 & 21 & 14.0 & 0.2 & 1.49 & 0.04 & 3.18 & 0.02 & 5.2 & 0.1 & 0 & 0 \\
\hline HD 43197 & 4.4 & 2.1 & 5457 & 33 & 0.75 & 0.02 & 1.00 & 0.02 & 4.46 & 0.03 & 0.97 & 0.03 & 0 & 0 \\
\hline HD 43691 & 2.1 & 1.7 & 6101 & 67 & 3.2 & 0.1 & 1.33 & 0.03 & 4.14 & 0.03 & 1.6 & 0.1 & 0 & 0 \\
\hline HD 44219 & 9.7 & 0.8 & 5739 & 50 & 1.82 & 0.02 & 1.01 & 0.01 & 4.16 & 0.02 & 1.37 & 0.03 & 0 & 0 \\
\hline HD 45350 & 7.1 & 0.9 & 5683 & 35 & 1.43 & 0.02 & 1.06 & 0.01 & 4.27 & 0.02 & 1.24 & 0.02 & 0 & 0 \\
\hline HD 45364 & 5.8 & 2.4 & 5523 & 28 & 0.575 & 0.004 & 0.86 & 0.02 & 4.53 & 0.02 & 0.83 & 0.01 & 0 & 0 \\
\hline HD 45652 & 5.4 & 2.7 & 5342 & 31 & 0.61 & 0.01 & 0.94 & 0.02 & 4.49 & 0.03 & 0.91 & 0.02 & 0 & 0 \\
\hline HD 46375 & 11.9 & 1.1 & 5379 & 19 & 0.77 & 0.01 & 0.91 & 0.01 & 4.38 & 0.01 & 1.01 & 0.01 & 1 & 0 \\
\hline $\mathrm{HD}$ & 5.3 & 0.8 & 5729 & 24 & 9 & 0.02 & 1.05 & 0.01 & 6 & 0.02 & 1.11 & 0.02 & 0 & 0 \\
\hline HD 49674 & 3.6 & 0.8 & 5655 & 25 & 0.99 & 0.02 & 1.06 & 0.01 & 4.42 & 0.01 & 1.04 & 0.02 & 0 & 0 \\
\hline HD 50499 & 2.3 & 0.4 & 6112 & 30 & 2.26 & 0.04 & 1.27 & 0.01 & 4.28 & 0.02 & 1.34 & 0.03 & 0 & 0 \\
\hline HD 50554 & 2.1 & 0.5 & 6047 & 17 & 1.37 & 0.01 & 1.1 & 0.01 & 4.41 & 0.01 & 1.07 & 0.01 & 0 & 0 \\
\hline HD 52265 & 2.2 & 0.7 & 6183 & 41 & 2.06 & 0.03 & 1.22 & 0.01 & 4.32 & 0.02 & 1.25 & 0.02 & 0 & 0 \\
\hline HD 5319 & 6.1 & 1.4 & 4888 & 39 & 8.2 & 0.1 & 1.2 & 0.1 & 3.3 & 0.04 & 4.0 & 0.1 & 0 & 0 \\
\hline HD 5608 & 3.0 & 0.3 & 4897 & 25 & 13.1 & 0.3 & 1.5 & 0.04 & 3.2 & 0.02 & 5.0 & 0.1 & 0 & 0 \\
\hline HD 5891 & 5.7 & 1.5 & 4796 & 41 & 39.1 & 0.4 & 1.1 & 0.1 & 2.57 & 0.05 & 9.1 & 0.2 & 0 & 0 \\
\hline HD 60532 & 3.0 & 0.2 & 6188 & 17 & 9.3 & 0.1 & 1.46 & 0.03 & 3.75 & 0.02 & 2.66 & 0.03 & 0 & 0 \\
\hline HD 63454 & 2.4 & 3.1 & 4787 & 12 & 0.24 & 0.01 & 0.79 & 0.01 & 4.62 & 0.02 & 0.72 & 0.01 & 0 & 0 \\
\hline HD 63765 & 7.9 & 3.1 & 5474 & 35 & 0.58 & 0.01 & 0.85 & 0.02 & 4.51 & 0.03 & 0.85 & 0.02 & 0 & 0 \\
\hline HD 6434 & 12.2 & 0.7 & 5907 & 21 & 1.208 & 0.003 & 0.83 & 0.01 & 4.31 & 0.01 & 1.05 & 0.01 & 0 & 0 \\
\hline HD 65216 & 4.6 & 3.1 & 5694 & 45 & 0.72 & 0.02 & 0.91 & 0.03 & 4.51 & 0.04 & 0.87 & 0.02 & 1 & 0 \\
\hline HD 66428 & 5.9 & 0.8 & 5721 & 29 & 1.28 & 0.02 & 1.06 & 0.01 & 4.33 & 0.02 & 1.15 & 0.02 & 0 & 0 \\
\hline HD 6718 & 6.2 & 2.0 & 5805 & 46 & 1.06 & 0.02 & 0.97 & 0.02 & 4.4 & 0.03 & 1.02 & 0.03 & 0 & 0 \\
\hline HD 68988 & 2.1 & 0.5 & 5880 & 21 & 1.34 & 0.02 & 1.15 & 0.01 & 4.39 & 0.01 & 1.12 & 0.02 & 0 & 0 \\
\hline HD 69830 & 10.4 & 2.5 & 5401 & 28 & 0.59 & 0.01 & 0.85 & 0.02 & 4.47 & 0.02 & 0.88 & 0.02 & 0 & 0 \\
\hline HD 70642 & 3.6 & 0.9 & 5675 & 18 & 0.92 & 0.01 & 1.02 & 0.01 & 4.45 & 0.01 & 0.99 & 0.01 & 0 & 0 \\
\hline HD 7199 & 9.2 & 2.5 & 5357 & 39 & 0.72 & 0.01 & 0.93 & 0.02 & 4.41 & 0.03 & 0.99 & 0.02 & 0 & 0 \\
\hline HD 72659 & 6.4 & 0.7 & 5994 & 50 & 2.09 & 0.02 & 1.08 & 0.02 & 4.21 & 0.02 & 1.34 & 0.03 & 0 & 0 \\
\hline HD 73256 & 4.5 & 2.3 & 5514 & 35 & 0.75 & 0.02 & 0.98 & 0.03 & 4.47 & 0.03 & 0.95 & 0.02 & 0 & 0 \\
\hline HD 73267 & 11.8 & 1.4 & 5434 & 18 & 0.74 & 0.01 & 0.89 & 0.01 & 4.4 & 0.02 & 0.97 & 0.01 & 0 & 0 \\
\hline HD 73526 & 7.9 & 0.5 & 5669 & 53 & 2.18 & 0.01 & 1.09 & 0.01 & 4.1 & 0.02 & 1.53 & 0.03 & 0 & 0 \\
\hline HD 73534 & 7.1 & 0.8 & 4958 & 45 & 3.4 & 0.1 & 1.15 & 0.03 & 3.7 & 0.03 & 2.5 & 0.1 & 0 & 0 \\
\hline HD 74156 & 4.3 & 0.6 & 6070 & 56 & 3.08 & 0.03 & 1.24 & 0.04 & 4.12 & 0.03 & 1.59 & 0.04 & 0 & 0 \\
\hline HD 7449 & 2.2 & 1.3 & 6060 & 42 & 1.26 & 0.02 & 1.05 & 0.02 & 4.44 & 0.02 & 1.02 & 0.02 & 1 & 0 \\
\hline HD 75289 & 1.7 & 0.4 & 6143 & 25 & 1.97 & 0.01 & 1.23 & 0.01 & 4.33 & 0.01 & 1.24 & 0.01 & 1 & 0 \\
\hline HD 75898 & 3.8 & 0.6 & 6019 & 66 & 2.88 & 0.02 & 1.28 & 0.02 & 4.15 & 0.03 & 1.56 & 0.04 & 0 & 0 \\
\hline HD 76700 & 6.2 & 0.9 & 5706 & 41 & 1.73 & 0.03 & 1.13 & 0.02 & 4.22 & 0.03 & 1.35 & 0.03 & 0 & 0 \\
\hline HD 77338 & 7.8 & 3.4 & 5261 & 29 & 0.57 & 0.02 & 0.91 & 0.03 & 4.47 & 0.04 & 0.91 & 0.03 & 0 & 0 \\
\hline
\end{tabular}


Table A.1. continued.

\begin{tabular}{|c|c|c|c|c|c|c|c|c|c|c|c|c|c|c|}
\hline Star & $\begin{array}{r}t \\
(\mathrm{Gyr})\end{array}$ & $\begin{array}{r}\Delta t \\
(\mathrm{Gyr})\end{array}$ & $\begin{array}{l}T_{\text {eff }} \\
(\mathrm{K})\end{array}$ & $\begin{array}{r}\Delta T_{\text {eff }} \\
(\mathrm{K})\end{array}$ & $\begin{array}{r}L \\
\left(L_{\odot}\right) \\
\end{array}$ & $\begin{array}{r}\Delta L \\
\left(L_{\odot}\right)\end{array}$ & $\begin{array}{r}M \\
\left(M_{\odot}\right)\end{array}$ & $\begin{array}{r}\Delta M \\
\left(M_{\odot}\right)\end{array}$ & $\begin{array}{r}\log g \\
\left(\mathrm{~cm} / \mathrm{s}^{2}\right) \\
\end{array}$ & $\begin{array}{r}\Delta \log g \\
\left(\mathrm{~cm} / \mathrm{s}^{2}\right)\end{array}$ & $\begin{array}{r}R \\
\left(R_{\odot}\right) \\
\end{array}$ & $\begin{array}{r}\Delta R \\
\left(R_{\odot}\right)\end{array}$ & Bin & $\operatorname{Tr}$ \\
\hline HD 7924 & 5.4 & 2.6 & 5218 & 12 & 0.36 & 0.01 & 0.79 & 0.01 & 4.59 & 0.02 & 0.74 & 0.01 & 0 & 0 \\
\hline HD 79498 & 4.2 & 0.9 & 5741 & 20 & 1.11 & 0.02 & 1.05 & 0.01 & 4.4 & 0.02 & 1.07 & 0.02 & 1 & 0 \\
\hline HD 80606 & 2.7 & 0.7 & 5558 & 23 & 0.81 & 0.02 & 1.03 & 0.01 & 4.47 & 0.01 & 0.97 & 0.02 & 1 & 1 \\
\hline HD 81040 & 3.6 & 1.5 & 5678 & 24 & 0.79 & 0.02 & 0.96 & 0.02 & 4.49 & 0.02 & 0.92 & 0.02 & 0 & 0 \\
\hline HD 81688 & 6.3 & 2.9 & 4830 & 64 & 54 & 1 & 1.1 & 0.2 & 2.4 & 0.1 & 10.5 & 0.4 & 0 & 0 \\
\hline HD 82886 & 3.3 & 0.5 & 5083 & 38 & 13.5 & 0.2 & 1.3 & 0.1 & 3.21 & 0.04 & 4.8 & 0.1 & 0 & 0 \\
\hline HD 82943 & 3.1 & 0.4 & 5944 & 18 & 1.54 & 0.02 & 1.14 & 0.01 & 4.35 & 0.01 & 1.17 & 0.02 & 0 & 0 \\
\hline HD 83443 & 5.2 & 1.9 & 5458 & 28 & 0.76 & 0.02 & 0.99 & 0.02 & 4.45 & 0.03 & 0.98 & 0.02 & 0 & 0 \\
\hline HD 8535 & 3.3 & 0.5 & 6142 & 34 & 1.92 & 0.01 & 1.15 & 0.01 & 4.31 & 0.02 & 1.23 & 0.02 & 0 & 0 \\
\hline HD 85390 & 6.8 & 2.9 & 5174 & 17 & 0.39 & 0.01 & 0.81 & 0.02 & 4.56 & 0.02 & 0.78 & 0.01 & 0 & 0 \\
\hline HD 85512 & 8.2 & 3.0 & 4530 & 8 & 0.138 & 0.002 & 0.64 & 0.01 & 4.67 & 0.01 & 0.6 & 0.01 & 0 & 0 \\
\hline HD 8574 & 4.4 & 0.6 & 6092 & 56 & 2.35 & 0.04 & 1.17 & 0.02 & 4.22 & 0.03 & 1.38 & 0.04 & 0 & 0 \\
\hline HD 86081 & 5.5 & 0.9 & 5887 & 56 & 2.51 & 0.02 & 1.18 & 0.04 & 4.14 & 0.03 & 1.53 & 0.03 & 0 & 0 \\
\hline HD 86264 & 0.8 & 0.2 & 6616 & 39 & 4.02 & 0.04 & 1.46 & 0.01 & 4.23 & 0.02 & 1.53 & 0.02 & 0 & 0 \\
\hline HD 87883 & 7.5 & 3.8 & 4971 & 22 & 0.327 & 0.004 & 0.81 & 0.02 & 4.56 & 0.02 & 0.77 & 0.01 & 0 & 0 \\
\hline HD 88133 & 5.7 & 0.3 & 5468 & 25 & 3.4 & 0.1 & 1.23 & 0.02 & 3.9 & 0.02 & 2.1 & 0.1 & 0 & 0 \\
\hline HD 89307 & 4.6 & 1.7 & 6011 & 59 & 1.34 & 0.03 & 1.02 & 0.03 & 4.38 & 0.03 & 1.07 & 0.03 & 0 & 0 \\
\hline HD 89744 & 2.5 & 0.3 & 6270 & 54 & 6.29 & 0.01 & 1.49 & 0.02 & 3.95 & 0.02 & 2.13 & 0.04 & 1 & 0 \\
\hline HD 90156 & 5.7 & 1.7 & 5721 & 28 & 0.74 & 0.01 & 0.89 & 0.02 & 4.49 & 0.02 & 0.88 & 0.01 & 0 & 0 \\
\hline HD 92788 & 4.2 & 1.1 & 5788 & 38 & 1.28 & 0.02 & 1.09 & 0.02 & 4.36 & 0.02 & 1.13 & 0.02 & 0 & 0 \\
\hline HD 93083 & 6.2 & 4.4 & 5025 & 21 & 0.35 & 0.02 & 0.83 & 0.02 & 4.57 & 0.03 & 0.78 & 0.02 & 0 & 0 \\
\hline HD 9446 & 3.7 & 2.0 & 5790 & 45 & 1.06 & 0.03 & 1.04 & 0.03 & 4.43 & 0.03 & 1.03 & 0.03 & 0 & 0 \\
\hline HD 95089 & 3.0 & 0.2 & 4952 & 19 & 13.0 & 0.1 & 1.48 & 0.04 & 3.22 & 0.02 & 4.9 & 0.1 & 0 & 0 \\
\hline HD 96063 & 2.9 & 0.2 & 5073 & 19 & 12.3 & 0.1 & 1.42 & 0.03 & 3.27 & 0.01 & 4.5 & 0.1 & 0 & 0 \\
\hline HD 96127 & 7.2 & 2.1 & 3943 & 34 & 516 & 22 & 1.0 & 0.1 & 1.1 & 0.1 & 48.8 & 1.9 & 0 & 0 \\
\hline HD 96167 & 4.7 & 0.6 & 5753 & 49 & 3.7 & 0.2 & 1.3 & 0.1 & 3.97 & 0.02 & 1.9 & 0.1 & 0 & 0 \\
\hline HD 97658 & 9.7 & 2.8 & 5211 & 16 & 0.35 & 0.01 & 0.74 & 0.01 & 4.58 & 0.02 & 0.73 & 0.01 & 0 & 1 \\
\hline HD 98219 & 3.2 & 0.4 & 4952 & 31 & 11.4 & 0.3 & 1.5 & 0.1 & 3.27 & 0.03 & 4.6 & 0.1 & 0 & 0 \\
\hline HD 99109 & 6.0 & 3.0 & 5270 & 24 & 0.56 & 0.02 & 0.93 & 0.02 & 4.49 & 0.03 & 0.9 & 0.03 & 0 & 0 \\
\hline HD 99492 & 8.1 & 3.2 & 4917 & 21 & 0.33 & 0.01 & 0.82 & 0.02 & 4.55 & 0.02 & 0.79 & 0.02 & 1 & 0 \\
\hline HD 99706 & 2.9 & 0.2 & 4847 & 17 & 15.6 & 0.1 & 1.53 & 0.03 & 3.12 & 0.02 & 5.6 & 0.1 & 0 & 0 \\
\hline HIP 14810 & 7.1 & 2.1 & 5570 & 47 & 0.99 & 0.03 & 1.00 & 0.02 & 4.37 & 0.04 & 1.07 & 0.04 & 0 & 0 \\
\hline HIP 5158 & 4.5 & 3.2 & 4571 & 14 & 0.19 & 0.01 & 0.75 & 0.01 & 4.63 & 0.02 & 0.69 & 0.02 & 0 & 0 \\
\hline HIP 57050 & 8.8 & 3.6 & 3542 & 2 & 0.0068 & $2.0 \mathrm{E}-4$ & 0.2 & 0.0 & 5.05 & 0.01 & 0.219 & 0.003 & 0 & 0 \\
\hline HIP 57274 & 8.9 & 2.1 & 4636 & 35 & 0.2 & 0.01 & 0.72 & 0.01 & 4.61 & 0.01 & 0.69 & 0.03 & 0 & 0 \\
\hline kappa CrB & 3.2 & 0.4 & 4899 & 30 & 11.8 & 0.2 & 1.5 & 0.1 & 3.24 & 0.03 & 4.8 & 0.1 & 0 & 0 \\
\hline KELT-3 & 1.8 & 1.2 & 6413 & 55 & 3.3 & 0.1 & 1.3 & 0.02 & 4.2 & 0.01 & 1.5 & 0.1 & 0 & 1 \\
\hline KELT-6 & 2.6 & 2.8 & 6267 & 46 & 3.8 & 0.6 & 1.2 & 0.1 & 4.1 & 0.1 & 1.7 & 0.1 & 0 & 1 \\
\hline Kepler-21 & 3.7 & 0.4 & 6264 & 60 & 5.20 & 0.04 & 1.31 & 0.03 & 3.97 & 0.02 & 1.94 & 0.04 & 0 & 1 \\
\hline Kepler-37 & 3.3 & 0.6 & 5630 & 9 & 0.559 & 0.002 & 0.85 & 0.01 & 4.57 & 0.01 & 0.787 & 0.004 & 0 & 1 \\
\hline Kepler-68 & 6.1 & 0.5 & 5868 & 32 & 1.62 & 0.03 & 1.07 & 0.01 & 4.28 & 0.01 & 1.23 & 0.03 & 0 & 1 \\
\hline mu Ara & 5.4 & 0.7 & 5817 & 44 & 1.78 & 0.04 & 1.14 & 0.02 & 4.25 & 0.03 & 1.32 & 0.04 & 0 & 0 \\
\hline NGC 24233 & 6.7 & 3.0 & 4446 & 26 & 73 & 14 & 1.2 & 0.2 & 2.19 & 0.01 & 14.4 & 1.6 & 0 & 0 \\
\hline Qatar-1 & 6.9 & 3.8 & 4730 & 15 & 0.24 & 0.01 & 0.78 & 0.02 & 4.59 & 0.02 & 0.73 & 0.01 & 0 & 1 \\
\hline tau Boo & 1.8 & 0.4 & 6408 & 45 & 3.11 & 0.04 & 1.33 & 0.01 & 4.24 & 0.02 & 1.43 & 0.03 & 1 & 0 \\
\hline TrES-1 & 9.5 & 3.1 & 5492 & 57 & 0.77 & 0.02 & 0.91 & 0.03 & 4.42 & 0.04 & 0.97 & 0.03 & 0 & 1 \\
\hline TrES-2 & 5.0 & 1.0 & 5958 & 65 & 1.7 & 0.1 & 1.09 & 0.03 & 4.3 & 0.01 & 1.2 & 0.1 & 0 & 1 \\
\hline TrES-3 & 7.9 & 1.4 & 5614 & 29 & 0.77 & 0.01 & 0.9 & 0.02 & 4.45 & 0.01 & 0.93 & 0.02 & 0 & 1 \\
\hline TrES-4 & 2.1 & 0.1 & 6327 & 25 & 3.42 & 0.04 & 1.37 & 0.01 & 4.192 & 0.004 & 1.54 & 0.02 & 0 & 1 \\
\hline TrES-5 & 7.5 & 3.7 & 5087 & 31 & 0.41 & 0.01 & 0.85 & 0.03 & 4.53 & 0.03 & 0.82 & 0.02 & 0 & 1 \\
\hline WASP-11 & 5.7 & 2.1 & 4917 & 18 & 0.29 & 0.01 & 0.79 & 0.01 & 4.59 & 0.01 & 0.74 & 0.02 & 0 & 1 \\
\hline WASP-14 & 1.5 & 1.4 & 6454 & 52 & 4.2 & 0.5 & 1.33 & 0.04 & 4.13 & 0.03 & 1.7 & 0.1 & 0 & 1 \\
\hline WASP-16 & 8.5 & 1.2 & 5633 & 49 & 1.09 & 0.04 & 0.98 & 0.02 & 4.34 & 0.01 & 1.1 & 0.04 & 0 & 1 \\
\hline WASP-18 & 0.9 & 0.2 & 6167 & 7 & 1.7 & 0.04 & 1.2 & 0.001 & 4.39 & 0.01 & 1.15 & 0.02 & 0 & 1 \\
\hline WASP-19 & 3.6 & 1.8 & 5526 & 39 & 0.76 & 0.01 & 1.00 & 0.02 & 4.47 & 0.02 & 0.95 & 0.02 & 0 & 1 \\
\hline WASP-2 & 0.7 & 0.3 & 5345 & 8 & 0.47 & 0.01 & 0.9 & 0.001 & 4.581 & 0.004 & 0.8 & 0.01 & 0 & 1 \\
\hline WASP-21 & 3.7 & 0.7 & 6123 & 38 & 1.43 & 0.03 & 1.03 & 0.02 & 4.39 & 0.01 & 1.06 & 0.02 & 0 & 1 \\
\hline WASP-25 & 3.2 & 1.2 & 5582 & 21 & 0.72 & 0.02 & 0.97 & 0.01 & 4.5 & 0.01 & 0.91 & 0.02 & 0 & 1 \\
\hline WASP-26 & 3.1 & 0.4 & 5881 & 13 & 1.26 & 0.01 & 1.09 & 0.01 & 4.4 & 0.01 & 1.08 & 0.01 & 0 & 1 \\
\hline WASP-34 & 6.8 & 1.3 & 5758 & 55 & 1.19 & 0.03 & 1.01 & 0.02 & 4.35 & 0.02 & 1.1 & 0.04 & 0 & 1 \\
\hline WASP-37 & 7.7 & 1.4 & 5956 & 55 & 1.1 & 0.04 & 0.9 & 0.02 & 4.39 & 0.02 & 0.99 & 0.04 & 0 & 1 \\
\hline WASP-39 & 6.7 & 2.4 & 5466 & 34 & 0.57 & 0.01 & 0.86 & 0.02 & 4.51 & 0.02 & 0.85 & 0.01 & 0 & 1 \\
\hline WASP-4 & 5.5 & 2.0 & 5435 & 31 & 0.6 & 0.01 & 0.91 & 0.02 & 4.51 & 0.02 & 0.88 & 0.01 & 0 & 1 \\
\hline WASP-41 & 3.9 & 1.0 & 5555 & 27 & 0.7 & 0.02 & 0.96 & 0.01 & 4.5 & 0.01 & 0.91 & 0.02 & 0 & 1 \\
\hline WASP-43 & 2.4 & 2.3 & 4756 & 12 & 0.215 & 0.002 & 0.75 & 0.01 & 4.64 & 0.01 & 0.68 & 0.01 & 0 & 1 \\
\hline
\end{tabular}


Table A.1. continued.

\begin{tabular}{|c|c|c|c|c|c|c|c|c|c|c|c|c|c|c|}
\hline Star & $\begin{array}{r}t \\
(\mathrm{Gyr})\end{array}$ & $\begin{array}{r}\Delta t \\
(\mathrm{Gyr}) \\
\end{array}$ & $\begin{array}{l}T_{\text {eff }} \\
(\mathrm{K})\end{array}$ & $\begin{array}{r}\Delta T_{\text {eff }} \\
(\mathrm{K}) \\
\end{array}$ & $\begin{array}{r}L \\
\left(L_{\odot}\right) \\
\end{array}$ & $\begin{array}{r}\Delta L \\
\left(L_{\odot}\right) \\
\end{array}$ & $\begin{array}{r}M \\
\left(M_{\odot}\right) \\
\end{array}$ & $\begin{array}{c}\Delta M \\
\left(M_{\odot}\right)\end{array}$ & $\begin{array}{r}\log g \\
\left(\mathrm{~cm} / \mathrm{s}^{2}\right) \\
\end{array}$ & $\begin{array}{r}\Delta \log g \\
\left(\mathrm{~cm} / \mathrm{s}^{2}\right)\end{array}$ & $\begin{array}{r}R \\
\left(R_{\odot}\right) \\
\end{array}$ & $\begin{array}{r}\Delta R \\
\left(R_{\odot}\right)\end{array}$ & Bin & $\operatorname{Tr}$ \\
\hline WASP-44 & 6.8 & 2.8 & 5402 & 31 & 0.6 & 0.02 & 0.9 & 0.02 & 4.49 & 0.03 & 0.89 & 0.02 & 0 & 1 \\
\hline WASP-48 & 9.1 & 0.8 & 5770 & 53 & 2.6 & 0.2 & 1.01 & 0.02 & 4.03 & 0.01 & 1.6 & 0.1 & 0 & 1 \\
\hline WASP-5 & 2.5 & 0.7 & 5611 & 14 & 0.79 & 0.02 & 1.01 & 0.01 & 4.49 & 0.01 & 0.94 & 0.01 & 0 & 1 \\
\hline WASP-52 & 3.8 & 2.6 & 5077 & 14 & 0.348 & 0.004 & 0.83 & 0.02 & 4.58 & 0.02 & 0.76 & 0.01 & 0 & 1 \\
\hline WASP-54 & 3.3 & 0.4 & 6090 & 26 & 1.61 & 0.02 & 1.1 & 0.01 & 4.36 & 0.01 & 1.14 & 0.02 & 0 & 1 \\
\hline WASP-58 & 12.6 & 0.1 & 5874 & 5 & 1.23 & 0.01 & 0.839 & 0.002 & 4.2936 & $1.0 \mathrm{E}-4$ & 1.073 & 0.005 & 0 & 1 \\
\hline WASP-60 & 8.8 & 1.4 & 5730 & 52 & 1.09 & 0.03 & 0.95 & 0.02 & 4.36 & 0.02 & 1.06 & 0.04 & 0 & 1 \\
\hline WASP-75 & 2.9 & 0.2 & 5862 & 9 & 1.1 & 0.01 & 1.051 & 0.004 & 4.435 & 0.002 & 1.02 & 0.01 & 0 & 1 \\
\hline WASP-8 & 4.2 & 1.1 & 5632 & 34 & 0.96 & 0.02 & 1.04 & 0.02 & 4.42 & 0.01 & 1.03 & 0.02 & 1 & 1 \\
\hline XO-2 & 9.6 & 0.9 & 5474 & 54 & 1.7 & 0.1 & 1.04 & 0.03 & 4.13 & 0.01 & 1.4 & 0.1 & 1 & 1 \\
\hline XO-3 & 1.7 & 1.0 & 6685 & 45 & 3.7 & 0.1 & 1.26 & 0.01 & 4.22 & 0.01 & 1.43 & 0.04 & 0 & 1 \\
\hline XO-5 & 12.4 & 0.6 & 5452 & 12 & 0.794 & 0.004 & 0.89 & 0.01 & 4.38 & 0.01 & 1.00 & 0.01 & 0 & 1 \\
\hline
\end{tabular}

